\title{
ON THE JACOBI SERIES
}

\author{
BY
}

\section{J. H. CURTISS}

1. Introduction. Let $F(z)$ denote a function regular in a neighborhood of each of the points of the complex $z$-plane determined by the $\lambda$ numbers $\alpha_{j}$, $j=1,2, \cdots, \lambda$, which are not necessarily distinct. By means of interpolation to $F(z)$ in the points $\alpha_{j}$, the coefficients $a_{n}$ of the following series are uniquely determined [20, p. 53]:

$$
\begin{array}{cl}
a_{0}+a_{1}\left(z-\alpha_{1}\right) \quad+a_{2}\left(z-\alpha_{1}\right)\left(z-\alpha_{2}\right)+\cdots+a_{\lambda-1}\left(z-\alpha_{1}\right) \cdots\left(z-\alpha_{\lambda-1}\right) \\
(1.1)+a_{\lambda} \omega(z)+a_{\lambda+1}\left(z-\alpha_{1}\right) \omega(z)+\cdots & +a_{2 \lambda-1}\left(z-\alpha_{1}\right) \cdots\left(z-\alpha_{\lambda-1}\right) \omega(z) \\
& +a_{2 \lambda}(\omega(z))^{2}+\cdots,
\end{array}
$$

where $\omega(z)=\prod_{j=1}^{\lambda}\left(z-\alpha_{j}\right)$. We shall denote the partial sums of this series by $S_{n}(z ; F)$ or $S_{n}(z), n=0,1,2, \cdots$, and the Cesàro means of order $r$ by $S_{n}^{(r)}(z ; F)$ or $S_{n}^{(r)}(z), n=0,1,2, \cdots$.

Jacobi [8](1) seems to have been the first to study developments of this type. He was interested in the problem of finding a formal expansion for $F(z)$ of the type $\sum_{0}^{\infty} q_{\nu}(z)(\omega(z))^{\nu}$, in which the functions $q_{\nu}(z)$ are polynomials of degree less than $\lambda$. The sum of the first $n$ terms of this original series of Jacobi is the polynomial of degree at most $\lambda n-1$ which interpolates to the function $F(z)$ in the points $\alpha_{j}$, each considered to be of multiplicity $n$. Thus the $n$th partial sum of Jacobi's original series is identical with the $\lambda n$th partial sum of the series (1.1). A change in the order of the points $\alpha_{j}$ naturally changes (1.1), but does not change Jacobi's original series. We shall call (1.1) the Jacobi series for $F(z)$ with respect to the points $\alpha_{j}$. The series is a generalization of the Taylor series, to which it reduces if the $\alpha_{j}$ all coincide.

The present study of the Jacobi series was undertaken at the suggestion of Professor J. L. Walsh. The purpose of the paper is to develop two general methods for studying the Jacobi series on the boundaries of its regions of convergence, and to obtain thereby certain typical results concerning the behavior of the series on these boundaries. The first method, in which the basic idea $(\$ 4)$ is due to Professor Walsh, consists in the study of certain expres-

Presented to the Society in two parts: $\$ \$ 1-5$ and 9, December 27,1934 , under the title The Jacobi interpolation series on the lemniscate of convergence (jointly with Professor J. L. Walsh); $\$ \$ 10-11$, October 30,1937 , under the title Uniform convergence and summability of the Jacobi series on an unrestricted lemniscate; received by the editors July 15, 1939, and, in revised form, June 13, 1940. The author is indebted to Professor Walsh not only for the contributions acknowledged in the text, but also for a number of suggestions concerning the exposition of the results contained in this paper.

(1) See also [20, pp. 54-64]; and [5, 6, 9, 10, 11, 12, 13, 19]. The Jacobi series has been used by Lebesgue to establish Weierstrass's theorem on approximation to continuous functions by polynomials; see [2, p. 60]. 
sions for the sums of the columns of the array (1.1). This method is developed in $\$ \S 4-7$, and is applied in $\S 8$ to the problem of determining the order of the coefficients of the series (1.1) under various conditions on the function $F(z)$. The second method consists in expressing the $\lambda n$th partial sum of (1.1) (that is, the $n$th partial sum of Jacobi's original series) as a definite integral similar to Dirichlet's integral ( $\$ 10)$. The technique of working with this integral is illustrated in $\$ 11$ by the derivation of several convergence tests for the Jacobi series analogous to the de la Vallée Poussin test and related tests for Fourier series. Both of the two general methods depend upon a fundamental connection between the Jacobi series and the Fourier series of the boundary values of $F(z)$ (Theorem 7.7). The existence of such boundary values is discussed in some detail in $\$ 7$, not only because it is an essential step in establishing the relation between the Jacobi and Fourier series, but also because it is a funcion-theoretic problem of some interest in itself.

Many further results concerning the convergence and summability of the Jacobi series are quite immediate consequences of the work in this paper. It is perhaps well to mention that one of the chief reasons for undertaking the present study was the hope that Jacobi series results might be indicative of those to be expected in the study of certain more general series of interpolation, and that the methods developed here might also be applicable in more general situations.

2. The regions of convergence. There exists a greatest positive number $\mu$ (finite or infinite) with the property that $F(z)$ is single-valued and regular on the set $D:|\omega(z)|<\mu[20$, p. 58]. This set consists of the finite plane if $\mu=\infty$. If $\mu<\infty$, it consists of $\lambda^{\prime}\left(1 \leqq \lambda^{\prime} \leqq \lambda\right)$ mutually exclusive Jordan regions $D_{k}$, $k=1, \cdots, \lambda^{\prime}$, the boundaries of which are contours $\left({ }^{2}\right)$ which we shall denote respectively by the letters $C_{k}, k=1, \cdots, \lambda^{\prime}$. The set $D_{k}+C_{k}$ will be denoted by $\bar{D}_{k}$, and the set $|\omega(z)| \leqq \mu$ by $\bar{D}$. The set $\sum_{1}^{\lambda^{\prime}} C_{k}$ is the lemniscate $\left.{ }^{3}\right) \Gamma$ : $|\omega(z)|=\mu$, which, for reasons contained in Theorem 2.1 below, is called the lemniscate of convergence of the series (1.1). In the neighborhood of a point $\beta$ on $\Gamma$ at which $d \omega / d z=\omega^{\prime}(z)$ has an $(m-1)$-fold zero, the locus $\Gamma$ consists of $m$ analytic arcs passing through the point $\beta$, with equally spaced tangents $\left({ }^{4}\right)$. The point $\beta$ is called a multiple point of order $m$ of $\Gamma$. By the index of a set $E$ on the lemniscate $\Gamma$, we shall mean the number $m(E)$ such that $\omega^{\prime}(z)$ has a zero of order $m(E)-1$ on $E$ but has no zero of higher order on $E$. We shall always denote the index of $\Gamma$ by $\bar{m}$.

The fundamental convergence theorem is as follows $[20$, pp. $57-60]\left({ }^{5}\right)$ :

(2) By a contour, we mean a Jordan curve of the finite plane composed of a finite number of analytic Jordan arcs. [21].

(3) For a complete discussion of lemniscates, the reader is referred to [20, pp. 54-56], and

(4) This statement is easily proved by the implicit function theorem.

(5) See also [10] and [11]. 
THEOREM 2.1. The series (1.1) converges absolutely for $z$ on $D$, and uniformly to $F(z)$ for $z$ on any closed limited subset of $D$. The series diverges for $|\omega(z)|>\mu$. Moreover, we have

$$
\begin{aligned}
\limsup _{n \rightarrow \infty}\left|a_{n}\right|^{1 / n} & =(1 / \mu)^{1 / \lambda}, \\
\left|F(z)-S_{n}(z)\right| & \leqq M\left(\mu_{1} / \mu_{2}\right)^{n / \lambda}, \\
|\omega(z)| & \leqq \mu_{1}<\mu,
\end{aligned}
$$

where $M$ is independent of $n$ and $z$ but not of $\mu_{1}$ and $\mu_{2}$, and where $\mu_{2}>\mu_{1}$ is an arbitrary positive number less than $\mu$.

The coefficients $a_{n}$ are given by the formulas $\left({ }^{6}\right)$

$$
\begin{aligned}
a_{\lambda \nu+K}=\frac{1}{2 \pi i} \int_{\Gamma^{\prime}} \frac{F(z) d z}{\left(z-\alpha_{1}\right)\left(z-\alpha_{2}\right) \cdots\left(z-\alpha_{K+1}\right)[\omega(z)]_{\nu}}, \\
K=0,1, \cdots, \lambda-1, \nu=0,1,2, \cdots,
\end{aligned}
$$

where $\Gamma^{\prime}$ denotes the lemniscate $|\omega(z)|=\mu_{1}$. It is easily shown that if by any means whatsoever we can find a series of type (1.1) which converges to $F(z)$ at every point $z$ for which $|\omega(z)|<\mu_{2}$, then the coefficients of this series (which must converge uniformly, $\left.|\omega(z)| \leqq \mu_{1}<\mu_{2}\right)$ are identical with the coefficients of (1.1) determined by interpolation. We shall make constant use of this remark in constructing examples, most of which will be derived from the binomial theorem.

3. Examples. An interesting special case is that in which $\lambda=2, \alpha_{1}=1$, $\alpha_{2}=-1$; the series (1.1) then has the form

$$
\sum_{\nu=0}^{\infty}\left[a_{2 v}\left(z^{2}-1\right)^{\nu}+a_{2 v+1}(z-1)\left(z^{2}-1\right)^{\nu}\right] .
$$

We shall frequently have occasion to refer to the following examples in the sequel.

(a) Let $g_{1}(z, q), q$ real, be an even function which, for $R z>0$, coincides with a branch of the function $z^{-2 q}$ chosen so that it is regular for $R z>0$ and so that $g_{1}(1, q)=1$.

In particular,

$$
g_{1}\left(z, \frac{1}{2}\right)=\left\{\begin{aligned}
1 / z, & R z>0 \\
-1 / z, & R z<0 .
\end{aligned}\right.
$$

We may write $\left({ }^{7}\right)$

(6) Integrals such as this one with complex differentials are to be taken in the LebesgueStieltjes sense, and those with real differentials are to be taken in the Lebesgue sense. See [16], especially pp. 64-67, for the theory of such integrals.

( ${ }^{7}$ With proper interpretation of the second member of the equation. 


$$
g_{1}(z, q)=\frac{1}{\left[1+\left(z^{2}-1\right)\right]^{q}}=\sum_{\nu=0}^{\infty} C_{\nu}^{-q}\left(z^{2}-1\right)^{\nu}, \quad\left|z^{2}-1\right|<1,
$$

where $C_{\nu}^{-q}=(-q)(-q-1) \cdots(-q-\nu+1) / \nu !=O\left(n^{q-1}\right), C_{0}^{-q}=1$.

(b) Let $g_{2}(z, q), q$ real, be an odd function which, for $R z>0$, coincides with a branch of the function $z^{1-2 q}$ chosen so that it is regular for $R z>0$ and so that $g_{2}(1, q)=1$.

In particular,

We may write $\left({ }^{7}\right)$

$$
g_{2}\left(z, \frac{1}{2}\right)= \begin{cases}+1, & R z>0 \\ -1, & R z<0\end{cases}
$$

$$
\begin{aligned}
g_{2}(z, q) & =\frac{1}{\left[1+\left(z^{2}-1\right)\right]^{q}}+\frac{z-1}{\left[1+\left(z^{2}-1\right)\right]^{q}} \\
& =\sum_{\nu=0}^{\infty}\left[C_{\nu}^{-q}\left(z^{2}-1\right)^{\nu}+C_{\nu}^{-q}(z-1)\left(z^{2}-1\right)^{\nu}\right], \quad\left|z^{2}-1\right|<1 .
\end{aligned}
$$

4. Walsh's representation of $F(z)$ by columns of (1.1). It was observed by Professor Walsh ( $\left.{ }^{8}\right)$ that for $\left|z^{2}-1\right|<\mu$, the series (3.1) expresses the generating function as the sum of two components, the first of which is an even function, and the second, the product of an even function into $(z-1)$. $\mathrm{He}$ found that if we write $\Psi_{0}(z) \equiv[F(z)+F(-z)] / 2+[F(z)-F(-z)] / 2 z$ and $\Psi_{1}(z) \equiv[F(z)-F(-z)] / 2 z$, then $F(z)=\Psi_{0}(z)+(z-1) \Psi_{1}(z)$ and $\Psi_{0}(z)=\sum_{0}^{\infty} a_{2 v}\left(z^{2}-1\right)^{\nu}, \Psi_{1}(z)=\sum_{0}^{\infty} a_{2 v+1}\left(z^{2}-1\right)^{\nu},\left|z^{2}-1\right|<\mu$. (Thus in $\S 3(\mathrm{~b})$, $\Psi_{0}=\left(1+z^{2}-1\right)^{-q}=\Psi_{1}$.)

He further pointed out that an analogous situation exists in the general case. We may write the following equation for $|\omega(z)|<\mu_{1}$ :

$$
\begin{aligned}
\Psi_{K}(z) & \equiv \sum_{\nu=0}^{\infty} a_{\lambda \nu+K}[\omega(z)]^{\nu} \\
& =\frac{1}{2 \pi i} \int_{\Gamma^{\prime}} \frac{F\left(z^{\prime}\right)}{\left(z^{\prime}-\alpha_{1}\right) \cdots\left(z^{\prime}-\alpha_{K+1}\right)} \sum_{0}^{\infty}\left[\frac{\omega(z)}{\omega\left(z^{\prime}\right)}\right]^{\nu} d z^{\prime} \\
& =\frac{1}{2 \pi i} \int_{\Gamma} \frac{F\left(z^{\prime}\right) P_{K}\left(z^{\prime}\right)}{\omega\left(z^{\prime}\right)-\omega(z)} d z^{\prime}, \quad K=0,1, \cdots, \lambda-1,
\end{aligned}
$$

where $P_{K}\left(z^{\prime}\right)=\prod_{j=K+2}^{\lambda}\left(z^{\prime}-\alpha_{j}\right), K=0,1, \cdots, \lambda-2$, and $P_{\lambda-1}\left(z^{\prime}\right)=1$. The equalities in (4.1) are easily established by reference to (2.1) and Theorem 2.1. We then have

(8) The material of this section was communicated to the author in conversations with Professor Walsh. 


$$
\begin{array}{rlrl}
F(z)= & \Psi_{0}(z)+\left(z-\alpha_{1}\right) \Psi_{1}(z)+\cdots \\
& +\left(z-\alpha_{1}\right)\left(z-\alpha_{2}\right) \cdots\left(z-\alpha_{\lambda-1}\right) \Psi_{\lambda-1}(z), \quad & |\omega(z)|<\mu_{1} .
\end{array}
$$

The functions $\Psi_{K}(z)$ are each invariant under the substitutions which leave $\omega(z)$ invariant, and in this sense are generalizations of even functions. Their Jacobi series are the second members of (4.1).

5. Evaluation of the integrals in (4.1). We henceforth shall assume that $\mu<\infty$. Let $w=\omega(z) / \mu$ (it is assumed invariably in the sequel that $z$ and $w$ are in this relationship) and let functions $z_{p}(w), p=1, \cdots, \lambda$, be defined by the identity

$$
\omega(z)-\mu w \equiv\left(z-z_{1}(w)\right)\left(z-z_{2}(w)\right) \cdots\left(z-z_{\lambda}(w)\right) .
$$

The functions $z_{p}(w)$ will be studied in some detail below; for the moment it suffices to observe that $\omega\left(z_{p}(w)\right) \equiv \mu w$, so that if $|w|<1$ (which will be the case if $z$ is in $D)$ then the points $z_{p}(w)$ are all in $D$.

If we write $\omega\left(z^{\prime}\right)-\omega(z)=\prod_{p=1}^{\lambda}\left(z^{\prime}-z_{p}(w)\right)$ in (4.1) and notice that $\omega^{\prime}\left(z_{h}(w)\right)=\prod_{p=1, p \neq h}^{\lambda}\left(z_{h}(w)-z_{p}(w)\right)$, then we obtain the following equation as a simple consequence of Cauchy's integral formula:

$$
\Psi_{K}(z)=\sum_{p=1}^{\lambda} \frac{F\left(z_{p}(w)\right) P_{K}\left(z_{p}(w)\right)}{\omega^{\prime}\left(z_{p}(w)\right)} \equiv \Phi_{K}(w), \quad K=0, \cdots, \lambda-1 .
$$

If the second members of (5.1) are replaced by their limiting values at poin ts $w$ for which $\omega^{\prime}\left(z_{p}(w)\right)=0, p=1, \cdots, \lambda$, then these formulas are valid for all values of $w$ such that $|w|<1$.

Equation (4.2) becomes

$$
\begin{aligned}
& F\left(z_{p}(w)\right)=\Phi_{0}(w)+\left(z_{p}(w)-\alpha_{1}\right) \Phi_{1}(w)+\cdots \\
& \quad+\left(z_{p}(w)-\alpha_{1}\right)\left(z_{p}(w)-\alpha_{2}\right) \cdots\left(z_{p}(w)-\alpha_{\lambda-1}\right) \Phi_{\lambda-1}(w), \quad|w|<1 .
\end{aligned}
$$

We find, by combining (4.1) and (5.1), that

$$
\Phi_{K}(w)=\sum_{\nu=0}^{\infty} a_{\lambda \nu+K}(\mu w)^{\nu}, \quad|w|<1, K=0,1, \cdots, \lambda-1 ;
$$

which implies that the functions $\Phi_{K}(w)$ are single-valued and regular for $|w|<1$, and that the series appearing in (5.3) are the Maclaurin series for these functions.

It is worth remarking that if $F\left(z_{p}(w)\right)=F\left(z_{p^{\prime}}(w)\right)$, for all $p$ and $p^{\prime}$, $p=1, \cdots, \lambda, p^{\prime}=1, \cdots, \lambda$, and for all $w$ on some point set $E$ having a limit point in the region $|w|<1$, then $F(z) \equiv \Psi_{0}(z)$. For the right member of (5.2) is formally a polynomial of degree $\lambda-1$ in the symbol $z_{p}(w)$, and at all but a finite number of points of $E$ the functions $z_{p}(w)$ will all be distinct (they obviously fail to be so only at points corresponding to points $z$ such 
that $\left.\omega^{\prime}(z)=0\right)$. At each point of $E$ at which the $z_{p}$ 's are distinct, we have $\Phi_{\lambda-1}(w)=0, \Phi_{\lambda-2}(w)=0, \cdots, \Phi_{1}(w)=0$; and it follows from the nature of $E$ and the regularity of the functions $\Phi_{K}$ that these equations must hold identically for $|w|<1$.

6. The functions $z_{p}(w)$. The functions $z_{p}(w)$ may be defined in such a way that they are algebraic functions $\left({ }^{9}\right)$ of a familiar type. It seems desirable at this point to give a somewhat detailed description of a Riemann surface for the function $w=\omega(z) / \mu$ which is adapted to our needs, and of certain properties of the inverse function on this surface.

Let the distinct roots of $\omega^{\prime}(z)$ be denoted by $\beta_{1}, \beta_{2}, \cdots, \beta_{\lambda_{1}}$, and their respective multiplicities by $m_{1}-1, m_{2}-1, \cdots, m_{\lambda_{1}}-1$. Let $\omega\left(\beta_{j}\right) / \mu=b_{j}$, $j=1, \cdots, \lambda_{1}$; these numbers $b_{j}$ are not necessarily distinct. We may assume that $b_{i} \neq 1, j=1, \cdots, \lambda_{1}$, for such a situation can always be brought about by introducing a rotative factor into the transformation $w=\omega(z) / \mu$, which would necessitate only trivial changes in the arguments used in the sequel. We construct the Riemann surface $R$ for this transformation as follows: The $w$-plane is replaced by $\lambda$ superimposed planes or sheets, and the points $b_{j}$, $j=1, \cdots, \lambda_{1}$, and the circle $\gamma:|w|=1$, are plotted in each plane. We connect points $b_{j}$ for which $\left|b_{j}\right| \geqq 1$ to $\infty$ by cuts which lie in the domains $|w| \geqq 1$ along rays starting at the origin and passing through these points $b_{j}$. There exists a number $\rho_{1}, 0 \leqq \rho_{1}<1$, such that the set of superimposed simply conected, annular-shaped regions $B$ bounded by the curves $\gamma,|w|=\rho_{1}$, and the segment $\rho_{1} \leqq w \leqq 1$, have the property that none of the points $b_{j}$ lie in the regions or on their boundaries except possibly on the circle $\gamma$. The points $b_{j}$ for which $\left|b_{j}\right|<\rho_{1}$ are connected by a cut through all the planes, lying along a Jordan arc which passes through each of these points and through the point $w=\rho_{1}$, and which coincides with the positive real axis from $w=\rho_{1}$ to $w=\infty$, but which does not pass through any point of $B$.

The function $z_{p}(w)$ is now defined in the usual way to be single-valued and regular on the $p$ th sheet of $R$ except at infinity and possibly at certain of the points $b_{j}$ on that sheet. The construction of $R$ is then completed by joining the edges of the planes across the cuts properly.

If $w \neq b_{j}, j=1, \cdots, \lambda_{1}$, the functions $z_{p}(w)$ have distinct values; but if $w=b_{j}$, exactly $m_{j}$ of these functions assume the common value $\beta_{j}$. All the functions are continuous for $w=b_{j}$, and the functions $z_{p}(w)$ for which $\omega^{\prime}\left(z_{p}\left(b_{j}\right)\right) \neq 0$ are regular for $w=b_{j}$. The group of $m_{j}$ functions which assume the value $\beta_{j}$ for $w=b_{j}$ forms a single cyclic system with respect to this value of $w$, because $[\partial / \partial w](\omega(z)-w) \neq 0\left[7\right.$, pp. 239-240]. For the moment let $b=b_{j}, \beta=\beta_{j}$, and $m=m_{j}$ for a fixed value of $j$; and let $z_{p_{1}}(b)=z_{p_{2}}(b)=\cdots=z_{p_{m}}(b)=\beta$. Then for $w$ in each of a set of $m$ regions $N_{h}$ : $|w-b| \leqq \delta>0$, located on the $m$ sheets numbered $p_{h}, h=1, \cdots, m$, there exists a development of the form

(9) See [1, chaps. 2-4], and [7, pp. 233-244]. 
$\beta+\sum_{\nu=1}^{\infty} c_{\nu}\left[(w-b)^{1 / m}\right]^{\nu}, c_{1} \neq 0$, which may be made to represent all the roots $z_{p_{1}}(w), \cdots, z_{p_{m}}(w)$, by giving to $(w-b)^{1 / m}$ all of its $m$ determinations $\left({ }^{10}\right)$. Thus letting $(w-b)_{h}^{1 / m}$ denote the determination for $z_{p_{h}}$, we have

$$
z_{p_{h}}(w)=\beta+\sum_{1}^{\infty} c_{\nu}\left[(w-b)_{h}^{1 / m}\right]^{\nu}, \quad c_{1} \neq 0, w \in N_{h} .
$$

Or again, we may write

$$
z_{p_{h}}(w)-\beta=(w-b)_{h}^{1 / m} A\left(W_{h}\right), \quad w \in N_{h},
$$

where $W_{h}=(w-b)_{h}^{1 / m}$ and $A\left(W_{h}\right)$ is a nonvanishing regular function of $W_{h}$ for $\left|W_{h}\right| \leqq \delta$. We have the inequalities ( $\left.{ }^{11}\right)$

$$
\begin{array}{ll}
M_{1} \leqq \frac{\left|z_{p_{h}}(w)-\beta\right|}{|w-b|^{1 / m}} \leqq M_{2}, & w \in N_{h}, w^{\prime} \in N_{h}, h=1, \cdots, m, \\
\frac{\left|z_{p_{h}}(w)-z_{p_{h}}\left(w^{\prime}\right)\right|}{\left|w-w^{\prime}\right|^{1 / m}} \leqq M_{3}, & w \in N_{h}, w^{\prime} \in N_{h}, h=1, \cdots, m .
\end{array}
$$

(The inequality (6.4) is easily proved by using the Heine-Borel theorem.)

The equation $z=z_{p}(w)$ gives a conformal one-to-one map of the annulus $B$ in the $p$ th sheet of $R$ onto a simply connected region $B_{p}$ in the $z$-plane. The region $B_{p}$ lies interior to one of the curves $C_{k}$ and is bounded by an arc of $C_{k}$, an analytic arc of the lemniscate $\Gamma_{\rho_{1}}:|w(z)|=\rho_{1}$, and analytic arcs of two orthogonal trajectories of $\Gamma$ (which may coincide) which are images of the segment $\rho_{1} \leqq w \leqq 1$. The regions $B_{p}, p=1, \cdots, \lambda$, have no point in common.

The correspondence of the boundaries of $B$ and of $B_{p}$ is one-to-one and continuous. Thus the set of functions $w=z_{p}\left(e^{i \theta}\right), p=1, \cdots, \lambda, 0 \leqq \theta \leqq 2 \pi$, gives a parametric representation of the lemniscate, and the arcs represented by the individual functions have no interior points in common.

Let us write $z_{p}\left(e^{i \theta}\right)=\zeta_{p}(\theta)$, and arrange the subscripts of the $\beta_{i}$ 's so that the $b_{j}$ 's for which $\left|b_{j}\right|=1$ are $b_{1}=e^{i \xi_{1}}, b_{2}=e^{i \xi_{2}}, \cdots, b_{s}=e^{i \xi_{s}}$, where $0<\xi_{1} \leqq \xi_{2} \leqq \cdots \leqq \xi_{8}<2 \pi$. It is obvious that we have constructed $R$ so that the subset of the functions $z_{p}(w)$ assigned to the curve $C_{k}$ forms a single cyclic system for $|w|=1$. Suppose that the $\kappa_{k}$ functions $z_{p_{1}}, z_{p_{2}}, \cdots, z_{p_{\alpha_{k}}}$ are assigned to $C_{k}$, and that $\zeta_{p_{i}}(\theta) \rightarrow \zeta_{p_{i+1}}(0), i=1, \cdots, \kappa_{k}-1$, and $\zeta_{p_{k_{k}}}(\theta) \rightarrow \zeta_{p_{1}}(0)$ as $\theta \rightarrow 2 \pi, \theta<2 \pi$. We now extend the range of definition of the functions $\zeta_{p_{i}}(\theta)$ by means of the following conventions:

(10) $\left[1\right.$, pp. 32-33], [7, pp. 238-240]. The reason why $c_{1} \neq 0$ in our case is brought out in the latter reference.

(11) Letters $M, M_{1}, M_{2}, \cdots$ will always denote finite positive constants which may depend on $\Gamma$ and on $F(z)$, but which will not depend directly upon any other apparent variable or subscript, unless the contrary is implied by the use of functional notation, as in the statement of Lemma 7.1. The significance of these letters will vary with the context. 


$$
\zeta_{p_{i}}(\theta \pm 2 n \pi)=\zeta_{p_{i+n}}(\theta), \quad i=1, \cdots, \kappa_{k}, n=1,2, \cdots,
$$

where $p_{\nu^{\prime}}=p_{\nu}$ for $\nu^{\prime} \equiv \nu\left(\bmod \kappa_{k}\right)$ and $0 \leqq \theta \leqq 2 \pi$. Then any one of the functions $\zeta_{p_{i}}(\theta)$, with its range of definition extended in this manner, will give a continuous parametric representation of the entire curve $C_{k}$, if $\theta$ is allowed to vary through $2 \pi \kappa_{k}$ radians instead of $2 \pi$ radians. That is, the function $z=\zeta_{p_{i}}(\theta)$ now sets up a one-to-one continuous correspondence between the points of $C_{k}$ and the points of a circumference on $R$, considered as $m$-fold, closed, and of length $2 \pi \kappa_{k}$. Any interval of values of $\theta$ of length $2 \pi \kappa_{k}$ may be used to obtain the representation. Thus $\zeta_{p_{i}}(\theta)$ is now a periodic function of $\theta$, the period being $2 \pi \kappa_{k}$. The function is an analytic function of $\theta$ for all values of this variable except possibly at certain values which satisfy the congruences $\theta \equiv \xi_{j}(\bmod 2 \pi), j=1, \cdots, s$.

Whenever our notation implies that $\theta$ is not restricted to the interval $[0,2 \pi]$-as, for instance, in the remainder of this section and in the lemmas of $\$ 11-$ it is to be understood that we are using the functions $\zeta_{p}(\theta)$ in the extended sense.

Suppose now that for a given $j$ we have $\zeta_{p_{1}}(\xi)=\zeta_{p_{2}}(\xi)=\cdots=\zeta_{p_{m j}}(\xi)=\beta_{j}$, where $\xi \equiv \xi_{j}(\bmod 2 \pi)$. Let $\beta=\beta_{j}$ and $m=m_{j}$. For $|\theta-\xi| \leqq \pi / 4$, we write $e^{i \theta}-e^{i \xi}=\left|2 \sin \frac{1}{2}(\theta-\xi)\right| e^{i \psi(\theta)}$ where

$$
\psi(\theta)=\left\{\begin{array}{l}
(3 \pi+\theta+\xi) / 2, \theta \leqq \xi \\
(\pi+\theta+\xi) / 2, \quad \theta>\xi .
\end{array}\right.
$$

There exists a number $\delta^{\prime}, 0<\delta^{\prime} \leqq \frac{1}{4} \pi$, and a method of assigning subscripts $p_{1}$, $p_{2}, \cdots, p_{m}$ to the functions $z_{p}(w)$, such that, by using (6.2), we may write

$$
\begin{aligned}
& \zeta_{p_{h}}(\theta)-\beta=\left(e^{i \theta}-e^{i \xi}\right)_{h}^{1 / m} A\left(W_{h}\right) \\
& =\left|2 \sin \frac{1}{2}(\theta-\xi)\right| 1 / m e^{i(\psi(\theta)+2 h \pi) / m} \cdot A\left(W_{h}\right), \quad|\theta-\xi| \leqq \delta^{\prime},
\end{aligned}
$$

where

$$
W_{h}=\left(e^{i \theta}-e^{i \xi}\right)_{h}^{1 / m}
$$

and $A\left(W_{h}\right)$ is a nonvanishing regular function of $W_{h}$ for $|\theta-\xi| \leqq \delta^{\prime}$. The folowing inequalities are then true for $|\theta-\xi| \leqq \delta^{\prime},\left|\theta^{\prime}-\xi\right| \leqq \delta^{\prime}$ :

$$
\begin{array}{cl}
M_{1} \leqq \frac{\left|\zeta_{p_{h}}(\theta)-\beta\right|}{|\theta-\xi|^{1 / m}} \leqq M_{2}, & h=1, \cdots, m ; \\
\frac{\left|\zeta_{p_{h}}(\theta)-\zeta_{p_{h}}\left(\theta^{\prime}\right)\right|}{\left|\theta-\theta^{\prime}\right|^{1 / m}} \leqq M_{3}, & h=1, \cdots, m .
\end{array}
$$

An important consequence of the periodicity of the functions $\zeta_{p}(\theta)$ is that with each number $\xi_{j}$ we may associate a positive number $\delta_{j}, \delta_{j} \leqq \frac{1}{4} \pi$, with the following property: If for any $p, 1 \leqq p \leqq \lambda$, we have $\zeta_{p}(\xi)=\beta_{j}$, where $\xi$ is any 
number such that $\xi \equiv \xi_{j}(\bmod 2 \pi)$, then a representation similar to (6.5) (with $h$ suitably determined) and inequalities (6.6) and (6.7) hold for $\zeta_{p}(\theta)$, provided that $|\theta-\xi| \leqq \delta_{j}$. The $\delta_{j}$ is independent of $\dot{p}$ and $\xi$. We shall assume that $\delta_{j}=\delta_{j^{\prime}}$ if $\xi_{j}=\xi_{j^{\prime}}$, that $\xi_{j}+\delta_{j}<\xi_{j+1}-\delta_{j+1}$ if $\xi_{j} \neq \xi_{j+1}$, and that $\xi_{s}+\delta_{s}<2 \pi$, $\xi_{1}-\delta_{1}>0$. It follows from these assumptions that $\zeta_{p}(\theta) \neq \beta_{j^{\prime}}, j^{\prime} \neq j$, for $\xi-\delta_{j} \leqq \theta \leqq \xi+\delta_{j}$, where $\xi \equiv \xi_{j}(\bmod 2 \pi)$.

Suppose now that for a given value of $p$ and of $k$, the point $\zeta_{p}(\theta)$ lies on $C_{k}$. The length of the arc of $C_{k}$ described by the point $\zeta_{p}(\theta)$ when $\theta$ varies from $\theta_{1}$ to $\theta_{2}$ is given by the formula

$$
\int_{\theta_{1}}^{\theta_{2}}\left|\frac{d \zeta_{p}(\theta)}{d \theta}\right| d \theta=\int_{\theta_{1}}^{\theta_{2}}\left|\frac{\mu}{\omega_{p}(\theta)}\right| d \theta,
$$

where $\omega_{p}(\theta)=\omega^{\prime}\left(\zeta_{p}(\theta)\right)$. It is well known that $\Gamma$ is rectifiable, which implies that the integrals in (6.8) are convergent for all values of $\theta_{1}$ and $\theta_{2}$. We shall need the following more precise result $\left({ }^{12}\right)$ :

LEMMA 6.1. $\int_{\theta}^{\theta+t} \mu\left|\omega_{p}(\tau)\right|^{-1} d \tau=O\left(|t|^{1 / \tilde{m}}\right)$ uniformly for $\theta$ on any closed interval $[a, b]$, where $\tilde{m}$ is the index of the arc of $\Gamma$ whose equation is $z=\zeta_{p}(\theta)$, $a \leqq \theta \leqq b$.

We shall show that for each number $\xi \in[a, b]$, there exist positive numbers $M(\xi)$ and $\delta(\xi)$ independent of $\theta$ such that

$$
\int_{\theta}^{\theta+t} \mu\left|\omega_{p}(\tau)\right|^{-1} d \tau \leqq M(\xi)|t|^{1 / \tilde{m}}
$$

for $\theta$ in the interval $I(\xi): \xi-\delta(\xi) \leqq \theta \leqq \xi+\delta(\xi)$ and for $|t| \leqq \delta(\xi)$. The HeineBorel theorem will then establish the lemma for all values of $\theta$ in the interval $[a, b]$.

The function $\omega_{p}(\theta)=\lambda \prod_{h=1}^{\lambda_{1}}\left(\zeta_{p}(\theta)-\beta_{h}\right)^{m h-1}$ is a continuous function of $\theta$. If $\omega_{p}(\xi) \neq 0$, then $\left|\omega_{p}(\theta)\right|$ is bounded from zero in some neighborhood of $\xi$, and $\int_{\theta}^{\theta+t}\left|\omega_{p}(\tau)\right|^{-1} d \tau=O(|t|)$ uniformly for $\theta$ in some interval $I(\xi)$. On the other hand, suppose that $\zeta_{p}(\xi)=\beta_{j}$ and $\xi \equiv \xi_{j}(\bmod 2 \pi)$. Then for $|\theta-\xi| \leqq \delta_{j}$, $\zeta(\theta)$ satisfies an inequality similar to (6.6), and $\pi^{\prime}\left|\zeta_{p}(\theta)-\beta_{h}\right|^{m h-1}$ is bounded from zero, where $\pi^{\prime}=\prod_{h=1}^{\lambda_{1}}, h \neq j$. We have

$$
\begin{aligned}
\int_{\theta}^{\theta+t}\left|\omega_{p}(\tau)\right|^{-1} d \tau & \leqq M \int_{\theta}^{\theta+t}\left|\zeta_{p}(\tau)-\beta\right|^{1-m} d \tau \\
& \leqq M_{1} \int_{\theta}^{\theta+t}|\tau-\xi|^{(1-m) / m} d \tau=O\left(|t|^{1 / m}\right), \\
& |\theta-\xi| \leqq \delta_{j / 2},|t| \leqq \frac{1}{2} \delta_{j},
\end{aligned}
$$

(12) The letters $t$ and $\tau$ will always denote real variables. By $f(t)=O\left(|t|^{\alpha}\right)$, we mean that positive numbers $M$ and $T$ exist such that $|f(t)| \leqq M|t|^{\alpha}$ for $|t| \leqq T$. 
where $\beta=\beta_{j}$ and $m=m_{j}$. Since $\tilde{m}$ is not less than $m$, the proof is complete $\left({ }^{13}\right)$.

By the measure of a set on a curve $C_{k}$, we shall mean its linear Lebesgue measure. Thus the measure of $\dot{a}$ set $E^{\prime}$ on $C_{k}$ which corresponds under the transformation $z=\zeta_{p}(\theta)$ to a set $E$ of values of $\theta$, is given by the formula $\int_{E} \mu\left|\omega_{p}(\theta)\right|^{-1} d \theta$; in particular, if $m(E)=0$, then $m\left(E^{\prime}\right)=0$ [16, pp. 123-125].

7. The classes $H^{\alpha}$ and the existence of boundary values. We shall say that $F(z)$ belongs to the class $H_{\Gamma}^{\alpha}, \alpha>0$, or more briefly, $F(z) \in H_{\Gamma}^{\alpha}$, if $\left(^{14}\right)$

$$
\begin{array}{rlrl}
\int_{\Gamma_{\rho}}|F(z)|^{\alpha}|d z| & =\mu \sum_{p=1}^{\lambda} \int_{|w|=\rho} \frac{\left|F_{p}\right|^{\alpha}}{\left|\omega_{p}^{\prime}\right|}|d w|=\mu \rho \int_{0}^{2 \pi} \sum_{p=1}^{\lambda} \frac{\left|F_{p}\right|^{\alpha}}{\left|\omega_{p}^{\prime}\right|} d \theta \\
& \leqq M(F, \Gamma), & 0<\rho<1,
\end{array}
$$

where $\Gamma_{\rho}$ denotes the lemniscate $|\omega(z)|=\rho \mu$, and where $F_{p}=F\left(z_{p}(w)\right)$, $\omega_{p}^{\prime}=\omega^{\prime}\left(z_{p}(w)\right), w=\rho e^{i \theta}$, and $|d z|$ and $|d w|$ denote differentials of arc length on $\Gamma_{\rho}$ and on the circle $|w|=\rho$ respectively. The classes $H_{\Gamma}^{\alpha}$ are analogous to the well known classes of functions regular for $|w|<1$ which F. Riesz [14] called the classes $H^{\alpha}$. We shall write $H$ instead of $H^{1}$, and $H_{\Gamma}$ instead of $H_{\Gamma}^{1}$; and we shall use $H_{\Gamma}^{\infty}$ to denote the class of functions $F(z)$, each uniformly bounded in modulus, $z \in D$.

TheOREM 7.1. If $F(z) \in H_{\Gamma}^{\alpha}$, then $F(z) \in H_{\Gamma}^{\beta}, \beta \leqq \alpha$.

The theorem is a direct consequence of the definition of the integrals in (7.1).

THEOREM 7.2. If $F(z) \in H_{\Gamma}^{\alpha}$, then $\Phi_{K}(w) \in H^{\alpha^{\prime}}$, where

(a) $\alpha^{\prime}=\alpha$ for $\bar{m}=1,0^{*}<\alpha \leqq \infty$,

(b) $\alpha^{\prime}=\alpha$ for $\bar{m} \geqq 1, \alpha \leqq 1$,

(c) $\alpha^{\prime}<\bar{m} \alpha /[\alpha(\bar{m}-1)+1]$ for $\bar{m}>1,1<\alpha<\infty$,

(d) $\alpha^{\prime}<\bar{m} /(\bar{m}-1)$ for $\bar{m}>1, \alpha=\infty$.

(13) Dr. S. E. Warschawski has pointed out in a communication to the author that the situation in Lemma 6.1 is typical of a broad class of curves. He formulates a general statement as follows:

Let the boundary of a simply connected region $D$ contain a free arc $C$ which consists of a finite number of arcs $\gamma_{1}, \gamma_{2}, \cdots, \gamma_{n}$ with bounded curvature. Let the measure of the corner at the point $z_{k}$ where the two arcs $\gamma_{k-1}$ and $\gamma_{k}$ meet be $\pi / m_{k}$. If $z=z(w)$ maps the region $|w|<1$ conformally onto $D$, and if the arc $\theta_{1} \leqq \theta \leqq \theta_{2}$ on $|w|=1$ corresponds to $C$, then the arc length $s(\theta)$ of $C$ satisfies the condition $s(\theta+t)-s(\theta)=O(|t| 1 / m), m=\max m_{k}$, uniformly, $\theta_{1} \leqq \theta \leqq \theta_{2}$.

The result can be derived by reference to the results of Osgood and Taylor, these Transactions, vol. 14 (1913), p. 282, which reduce the problem to a simple computation of the type carried out in the proof of Lemma 6.1. Dr. Warschawski further observes that the condition that the $\gamma_{k}$ 's have bounded curvature can be replaced by weaker ones; e.g., the conditions required in Theorem 10 of his thesis (Mathematische Zeitschrift, vol. 35 (1932), p. 433).

Lemma 6.1 can be derived from Warschawski's result by mapping the regions $B$ onto the unit circle.

(14) The first two integrals are to be taken in the Lebesgue-Stieltjes (or Riemann-Stieltjes) sense. 
To prove the theorem, we note that the hypothesis, when taken with (7.1), implies that

$$
\int_{0}^{2 \pi}\left|\frac{F_{p}}{\omega_{p}^{\prime}}\right|^{\alpha} d \theta \leqq M, \quad \rho_{1} \leqq \rho<1, \alpha<\infty
$$

Now

$$
\begin{array}{rlrl}
\int_{0}^{2 \pi}\left|\Phi_{K}(w)\right|^{\alpha^{\prime}} d \theta & \leqq M_{1} \sum_{p=1}^{\lambda} \int_{0}^{2 \pi}\left|\frac{F_{p} \cdot p_{K}\left(z_{p}(w)\right)}{\omega_{p}^{\prime}}\right|^{\alpha^{\prime}} d \theta & \\
& \leqq M_{2} \sum_{p=1}^{\lambda} \int_{0}^{2 \pi}\left|\frac{F_{p}}{\omega_{p}^{\prime}}\right|^{\alpha^{\prime}} d \theta, & & \rho<1 .
\end{array}
$$

If $\bar{m}=1$ and $\alpha<\infty$, or in any case if $\alpha \leqq 1$, we may write the inequalities

$$
\begin{aligned}
\int_{0}^{2 \pi}\left|\frac{F_{p}}{\omega_{p}^{\prime}}\right|^{\alpha} d \theta & \leqq M_{3} \int_{0}^{2 \pi} \frac{\left|F_{p}\right| \alpha}{\left|\omega_{p}^{\prime}\right|}\left|\omega_{p}^{\prime}\right|^{1-\alpha} d \theta \\
& \leqq M_{4} \int_{0}^{2 \pi} \frac{\left|F_{p}\right| \alpha}{\left|\omega_{p}^{\prime}\right|} d \theta \leqq M_{5}, \quad \rho_{1} \leqq \rho<1,
\end{aligned}
$$

because if $\bar{m}=1,\left|\omega_{p}^{\prime}\right|$ is uniformly bounded from zero in the regions $B$ of $R$. Thus for these values of $\bar{m}$ and $\alpha$, we have shown that $\int_{0}^{2 \pi}\left|\Phi_{K}(w)\right| \alpha d \theta \leqq M_{6}$ for $\rho_{1} \leqq \rho<1$, and a similar inequality obviously holds true for $0<\rho<1\left({ }^{15}\right)$. The case $\alpha=\infty, \bar{m}=1$, is easily taken care of by examining the formulas (5.1).

Consider now case $(c)$. Here the proof depends upon a lemma.

Lemma 7.1. There exists a number $M(q)$ such that $\int_{0}^{2 \pi}\left|\omega_{p}^{\prime}\right|^{-q} d \theta \leqq M(q)$, $p=1, \cdots, \lambda, \rho_{1} \leqq \rho<1$, where $q$ is any number less than $\bar{m} /(\bar{m}-1), \bar{m} \geqq 1$.

The result is immediate if $\bar{m}=1$. If $\bar{m}>1$, the result is again obvious for $\rho_{1} \leqq \rho \leqq \rho_{2}<1$ if $\rho_{2}$ is chosen suitably. In this case we show that for each number $\xi$ in the interval $0 \leqq \xi \leqq 2 \pi$ there exist numbers $\delta(\xi)>0, \rho(\xi) \geqq \rho_{1}$, and $M(q, \xi)=M(\xi)$ such that

$$
\int_{\xi-\delta(\xi)}^{\xi+\delta(\xi)}\left|\omega_{p}^{\prime}\right|-q d \theta \leqq M(\xi)
$$

for $\rho(\xi) \leqq \rho<1$. Suppose first that $\omega^{\prime}\left(z_{p}\left(e^{i \xi}\right)\right) \neq 0$. Then there exists a neighborhood of the point $w=e^{i \xi}$ in which $\left|\omega_{p}^{\prime}\right|$ is bounded from zero, and the existence of $M(\xi), \rho(\xi)$, and $\delta(\xi)$ follows at once. If $z_{p}\left(e^{i \xi}\right)=\beta_{j}$, and $e^{i \xi}=b_{j}=b$, then according to (6.3) there is a neighborhood of the point $e^{i \xi}$ in which $\left|\omega_{p}^{\prime}\right|^{-q} \leqq M_{1}(\xi)|w-b|^{-(m-1) q / m}$, where $m=m_{j}$. It is quite easily shown by elementary methods that any branch of the functions $\left(w-e^{i \xi}\right)^{-\eta}, \eta<1$, which

(15) Indeed, it is well known that $\int_{0}^{2 \pi}\left|\Phi_{K}(w)\right|^{\alpha} d \theta$ increases steadily with $\rho[18$, p. 174]. 
is regular for $|w|<1$, belongs to the class $H\left({ }^{16}\right)$. The existence of $M(\xi), \rho(\xi)$, and $\delta(\xi)$ is now obvious again, provided that we choose $q<m /(m-1)$ $\leqq \bar{m} /(\bar{m}-1)$. The proof of the lemma is completed by referring to the HeineBorel theorem and by observing that $\int_{0}^{2 \pi}\left|\omega_{p}^{\prime}\right|^{-q} d \theta \leqq \int_{I}\left|\omega_{p}^{\prime}\right|^{-q} d \theta$, where $I$ is any set of overlapping intervals covering the interval $[0,2 \pi]$.

To return to the proof of the theorem, we use the Hölder inequality to write

$$
\begin{aligned}
\int_{0}^{2 \pi}\left|\frac{F_{p}}{\omega_{p}^{\prime}}\right|^{\alpha^{\prime}} d \theta & =\int_{0}^{2 \pi}\left\{\frac{\left|F_{p}\right| \alpha}{\left|\omega_{p}^{\prime}\right|}\right\}^{\alpha^{\prime} / \alpha} \cdot\left\{\left|\frac{1}{\omega_{p}^{\prime}}\right|^{q}\right\}^{\left(\alpha^{\prime}-\alpha^{\prime} / \alpha\right) / q} d \theta \\
& \leqq\left\{\int_{0}^{2 \pi} \frac{\left|F_{p}\right| \alpha}{\left|\omega_{p}^{\prime}\right|} d \theta\right\}^{\alpha^{\prime} / \alpha}\left\{\int_{0}^{2 \pi}\left|\frac{1}{\omega_{p}^{\prime}}\right|^{q} d \theta\right\}^{\left(\alpha^{\prime}-\alpha^{\prime} / \alpha\right) / q}
\end{aligned}
$$

for $\rho_{1} \leqq \rho<1$. In this inequality, we must have $\alpha^{\prime}<\alpha$ and $\left(\alpha^{\prime} / \alpha\right)+\left(\alpha^{\prime}-\alpha^{\prime} / \alpha\right) / q$ $=1$, or $\alpha^{\prime}=q \alpha /(q+\alpha-1)$. By referring to (7.2) and the lemma, we see that the third member of (7.4) is bounded for $\rho_{1} \leqq \rho<1$ if $q<\bar{m} /(\bar{m}-1)$; that is, if $\alpha^{\prime}<\bar{m} \alpha /[\alpha(\bar{m}-1)+1]$. The proof of this section of the theorem is then completed by using (7.3).

Case (d) follows immediately from (5.2) and the lemma. The proof of the theorem is now complete.

It is easily shown that the inequality in (d) cannot be replaced by an equality. An example is given by the function $g_{2}\left(z, \frac{1}{2}\right)$, which belongs to the class $H_{\Gamma}^{\infty}$, where $\Gamma$ is now the lemniscate $\left|z^{2}-1\right|=1$, and for which $\Phi_{0}(w)$ and and $\Phi_{1}(w)$ obviously belong to the classes $H^{\alpha^{\prime}}, \alpha^{\prime}<2$, but not to the class $H^{2}$.

Similarly, the inequality in (c) cannot be replaced by an equality. An example to prove this may be constructed as follows: Let $F(z) \equiv 0$ for $R z<0$, and let $F(z)$ denote for $\left|z^{2}-1\right|<1, R z>0$, a branch of the function $z^{-1 / 2}$ $\cdot\left[\log \left(e / z^{2}\right)\right]^{-3 / 4}$ regular in the simply connected region defined by these inequalities. Then $F(z) \in H_{\Gamma}^{2}$, where $\Gamma$ is again the lemniscate $\left|z^{2}-1\right|=1$; and $\Phi_{1}(w) \in H^{\alpha^{\prime}}, \alpha^{\prime}<4 / 3$, but $\Phi_{1}(w)$ does not belong to the class $H^{4 / 3}$. The reader will have no difficulty in supplying a proof.

When $F(z) \in H_{\Gamma}^{\alpha}$, it does not follow in general that $\Psi_{K}(z) \in H_{\Gamma}^{\alpha}$ (an example is again given by $\left.g_{2}\left(z, \frac{1}{2}\right)\right)$, but the following result is an immediate consequence of Theorem 7.2.

Theorem 7.3. If $F(z) \in H^{\alpha}$, then

$$
\int_{\mathrm{r}_{\rho}}\left|\Psi_{K}(z)\right| \alpha^{\prime}\left|\omega^{\prime}(z)\right||d z|<M, \quad K=0,1, \cdots, \lambda-1,
$$

for $0<\rho<1$, where $\alpha^{\prime}$ is given by the formulas in Theorem 7.2. $\frac{1}{2} \leqq \rho<1$.

${ }^{(16)}$ We have $\int_{0}^{2 \pi}\left|1+\rho^{2}-2 \rho \cos (\theta-\xi)\right|^{-\eta / 2} d \theta \leqq \int_{0}^{2 \pi}(2 \rho)^{-\eta / 2}|1-\cos (\theta-\xi)|^{-\eta / 2} d \theta<M(\eta)$, 
The next two theorems are in the nature of converses to Theorems 7.2 and 7.3.

Theorem 7.4. If $\Phi_{K}(w) \in H^{\alpha}, K=0,1, \cdots, \lambda-1$, then

$$
\int_{\Gamma_{\rho}}|F(z)| \alpha\left|\omega^{\prime}(z)\right||d z|<M
$$

for $0<\rho<1$.

Theorem 7.5. If $\Psi_{K}(z) \in H_{\Gamma}^{\alpha}, K=0,1, \cdots, \lambda-1$, then $F(z) \in H_{\Gamma}^{\alpha}$.

The proofs are easily supplied by referring to (4.2) and (5.2). The example $g_{1}\left(z, \frac{1}{2}\right)$ shows that in general the factor $\left|\omega^{\prime}(z)\right|$ cannot be omitted from the integrand in Theorem 7.4.

We now consider the existence of boundary values. We define an $S$-path ( $\left.{ }^{17}\right)$ of a Jordan region $T$ bounded by a contour $C$ to be an analytic Jordan arc terminating at a point $Z$ of $C$ and lying in a closed triangular subregion of the closed region $\bar{T}$, the boundary of which has a vertex at $Z$ but has no other point in common with $C$, and is at no point tangent to $C$.

THEOREM 7.6. If $F(z) \in H_{\Gamma}^{\alpha}$, there exist finite-valued functions $\Phi_{K}{ }^{*}(w)$ and $F^{*}(z)$ such that

(a) $\Phi_{K}(w) \rightarrow \Phi_{K}^{*}(W), K=0,1, \cdots, \lambda-1$, for almost every point $W$ for which $|W|=1$, as $w \rightarrow W$ along any $S$-path of the regions $|w|<1$;

(b) $F(z) \rightarrow F^{*}(Z)$ for almost every point $Z$ on $\Gamma$ as $z \rightarrow Z$ along any $S$-path of $D\left({ }^{18}\right)$;

(c) $F^{*}\left(z_{p}(w)\right)=\Phi_{0}^{*}(w)+\left(z_{p}(w)-\alpha_{1}\right) \Phi_{1}^{*}(w)+\cdots+\left(z_{p}(w)-\alpha_{1}\right)\left(z_{p}(w)-\alpha_{2}\right)$ ... $\left(z_{p}(w)-\alpha_{\lambda-1}\right) \Phi_{\lambda_{-1}}^{*}(w)$ for almost every point $w$ on the circle $\gamma$ in the pth sheet of $R$;

(d) for almost every w for which $|w|=1$

$$
\Phi_{K}^{*}(w)=\sum_{p=1}^{\lambda} \frac{F^{*}\left(z_{p}(w)\right) P_{K}\left(z_{p}(w)\right)}{\omega^{\prime}\left(z_{p}(w)\right)}, \quad K=0,1, \cdots, \lambda-1 ;
$$

(e) for $\alpha^{\prime}$ given by the formulas in Theorem 7.2,

$$
\int_{\Gamma}\left|F^{*}(z)\right| \alpha|d z|<\infty, \int_{\nu}\left|\Phi_{K}^{*}(w)\right| \alpha^{\prime}|d w|<\infty, K=0,1, \cdots, \lambda-1 .
$$

Part (a) is a consequence of Theorem 7.2 and a theorem of F. Riesz $[14]\left({ }^{19}\right)$.

(17) $S$ for Stolz.

(18) The implication here is that $F^{*}(z)$ may be $m$-valued at a multiple point of order $m$, but is single-valued at all ordinary points of $\Gamma$.

$\left({ }^{19}\right)$ See also [22, p. 162]. 
We temporarily define $F^{*}\left(z_{p}(w)\right.$ for $|w|=1$ by the second member of the equality in (c). Now if the point $z_{p}(w)$ traverses an $S$-path in $B_{p}$ terminating at $z_{p}(W)$ on $\Gamma$, then the point $w$ traverses an $S$-path of the region $B$ on the $p$ th sheet of $R$, terminating at the point $W$ on that sheet. (This is an immediate consequence of the mapping properties of the analy tic function $w=\omega(z) / \mu$.) The set of points on the circle $\gamma$ in the pth sheet of $R$ at which one or more of the limits in (a) fail to exist is of measure zero, and (as observed at the end of $\S 6$ ) the corresponding set $\sigma$ on $\Gamma$ is also of measure zero. It is now easily seen from (5.2) that the statement in (b) is true for all points $Z$ of $\Gamma$ which are not in $\sigma$, which are also boundary points of $B_{p}$, and for which $\omega(Z) / \mu \neq 1$. For convenience we may add the set of points $Z$ defined by the equation $\omega(Z) / \mu=1$ to the set of excepted points in (b), and since the reasoning of this paragraph applied to $S$-paths in all of the regions $B_{p}, p=1, \cdots, \lambda$, the proof of (b) is complete.

Part (d) is now a consequence of (5.1).

Part (e) is proved immediately by referring to (7.1), parts (a) and (b) of the present theorem, and the lemma of Fatou [16, p. 29]. The proof of Theorem 7.6 is complete.

Now that the existence of boundary values and the validity of the limit in part (b) of Theorem 7.6 has been established, we shall revise the definition of $F^{*}(z)$ as follows: On each curve $C_{k}, F^{*}(Z)$ shall be the unique limit approached by the given function $F(z)$ as $z \rightarrow Z$ along any $S$-path of $D_{k}$, at each point $Z$ of $C_{k}$ for which this limit exists and is finite. At all other points of $C_{k}$, we let $F^{*}(z)=0$. The function $F^{*}(z)$ so defined exists everywhere on $\Gamma$, is single-valued except at the multiple points, and coincides with the previously defined boundary value function wherever the latter exists.

It will be convenient henceforth to use the single symbol $F(z)$ to designate the complete function consisting of $F(z),|\omega(z)|<\mu$, and $F^{*}(z),|\omega(z)|=\mu$; and we shall use the symbols $\Phi_{K}(w), K=0,1, \cdots, \lambda-1$, in a similarly extended sense.

The case $\alpha=1$ is of especial importance because of this theorem:

THEOREM 7.7. If $F(z) \in H_{\Gamma}$, then the series $\sum_{v=0}^{\infty} a_{\lambda \nu+K} \mu^{\nu} e^{i v \theta}, K=0,1, \cdots$, $\lambda-1$, are the Fourier series respectively of the functions $\Phi_{K}\left(e^{i \theta}\right), K=0,1, \cdots$, $\lambda-1$.

This result is a consequence of Theorem 7.2, equation (5.3), and a theorem of F. and M. Riesz [15, p. 42], which states that our conclusion is a necessary and sufficient condition for $\Phi_{K}(w) \in H$.

Conversely, if it be known of a series of the form (1.1) that for some number $\mu$ the series $\sum a_{\lambda \nu+K} \mu^{\nu} e^{i \nu \theta}, K=0,1, \cdots, \lambda-1$ are all Fourier series, then there exist functions $\Phi_{K}(w) \in H, K=0,1, \cdots, \lambda-1$ defined by equation (5.3), with boundary values on the unit circle for which these series are respectively the Fourier series; and there exists a function $F(z)$ defined by equation (4.2), 
such that $F(z) \omega^{\prime}(z) \in H_{\Gamma}$ (where $\Gamma$ is the lemniscate $|\omega(z)|=\mu$ ) and for which the original series is the Jacobi series with respect to the points $\alpha_{j}\left({ }^{20}\right)$.

Henceforth we shall assume that $F(z) \in H_{\Gamma}$, unless a statement is made to the contrary. We shall also make these abbreviations: $F\left(z_{p}\left(e^{i \theta}\right)\right)=f_{p}(\theta)$, $\Phi_{K}\left(e^{i \theta}\right)=\phi_{K}(\theta), P_{K}\left(z_{p}\left(e^{i \theta}\right)\right)=p_{K, p}(\theta)$.

Theorem 7.7 and Theorem 7.6, parts (d) and (e) enable us to write $\left({ }^{21}\right)$

$$
\begin{aligned}
\mu^{\nu} a_{\lambda \nu+K} & =\frac{1}{2 \pi} \int_{0}^{2 \pi} \phi_{K}(\theta) e^{-i \nu \theta} d \theta \\
& =\frac{1}{2 \pi} \int_{0}^{2 \pi} e^{-i \nu \theta} \sum_{p=1}^{\lambda}\left(\frac{f_{p}(\theta) p_{K, p}(\theta)}{\omega_{p}(\theta)}\right) d \theta \\
& =\frac{1}{2 \pi} \sum_{p=1}^{\lambda} \int_{0}^{2 \pi} \frac{f_{p}(\theta) p_{K, p}(\theta)}{\omega_{p}(\theta)} e^{-i \nu \theta} d \theta \\
& =\frac{\mu^{\nu}}{2 \pi i} \int_{\Gamma} \frac{F(z) d z}{\left(z-\alpha_{1}\right) \cdots\left(z-\alpha_{K+1}\right)[\omega(z)]^{\nu}}, \quad K=0,1, \cdots, \lambda-1 .
\end{aligned}
$$

Equation (7.5) is valid for all integral values of $\nu$ if the first member is replaced by zero when $\nu$ is negative. In particular, if $\nu=-1$ and $K=\lambda-1$, we have

$$
\frac{1}{2 \pi i \mu} \int_{\Gamma} F(z) d z=\frac{1}{2 \pi} \int_{0}^{2 \pi} \phi_{\lambda-1}(\theta) e^{i \theta} d \theta=\frac{1}{2 \pi i} \int_{\gamma} \Phi(w) d w=0
$$

$\left[15\right.$, p. 42]. A function $F_{k}(z)$ identical with $F(z)$ in $\bar{D}_{k}$ and identically zero elsewhere, will also be of class $H_{\Gamma}$. We may accordingly apply to $F_{k}(z)$ the argument which led to (7.6), and we find that $\int_{\Gamma} F_{k}(z) d z=\int_{c_{k}} F(z) d z=0$. Also, by Cauchy's integral theorem, $\int_{C_{k}} S_{n}(z) d z=0$. Combining these remarks, we have

THEOREM 7.8. $\int_{C_{k}} F(z) d z=\lim _{n \rightarrow \infty} \int_{C_{k}} S_{n}(z) d z=0, k=1, \cdots, \lambda^{\prime}$.

8. The coefficients. We now present a few typical results concerning the order of magnitude of the quantities $\mu^{\nu}\left|a_{\lambda \nu+K}\right|, K=0, \cdots, \lambda-1, \nu=0,1$, $2, \cdots$.

Our first theorem follows immediately from (7.5).

THEOREM 8.1. If $|F(z)| \leqq M(F), z \in \Gamma$, then $\mu^{\nu}\left|a_{\lambda v+K}\right| \leqq M(F) M_{1}(\Gamma)$, $K=0,1, \cdots, \lambda-1, \nu=0,1,2, \cdots$, where $M_{1}(\Gamma)$ is independent of $F(z)$.

${ }^{(20)}$ Strictly speaking, we are using the symbol $H_{\Gamma}$ in a slightly extended sense here, because in the present instance the lemniscate $\Gamma$ need not be the lemniscate of convergence of the Jacobi series.

(21) See [16, pp. 36-38], for the theorem on change of variables in the Lebesgue-Stieltjes integral. 
Our second theorem is simply the Riemann-Lebesgue theorem $[22$, p. 18] applied to the Fourier coefficients of the functions $\phi_{K}(\theta)$ :

TheOREM 8.2. $\operatorname{Lim}_{\nu \rightarrow \infty} \mu^{v} a_{\lambda \nu+K}=0, K=0,1, \cdots, \lambda-1$.

Suppose now that $|F(z)|$ is uniformly bounded on $\Gamma$. Let $C_{p}(\delta), \delta>0$, denote the modulus of continuity [22, p. 17] of the periodic function $f_{p}(\theta)$, and let $C(\delta)$ denote for each $\delta$ the largest of the numbers $C_{p}(\delta), p=1, \cdots, \lambda$.

ThEOREM 8.3. $\left|\mu^{\nu} a_{\lambda \nu+K}\right|<M\left[C(\pi / \nu)+(\pi / \nu)^{1 / \bar{m}}\right], K=0,1, \cdots, \lambda-1$, $\nu=1,2, \cdots$.

For the proof, we write

$$
2 \pi \mu^{\nu} a_{\lambda \nu+K}=\frac{1}{2} \int_{0}^{2 \pi}\left[\phi_{K}(\theta)-\phi_{K}(\theta+\pi / \nu)\right] e^{-i \nu \theta} d \theta,
$$

and by reference to (7.5) we see that it is sufficient to show that the integrals

$$
\begin{array}{r}
I_{K, p}=\int_{0}^{2 \pi}\left|\frac{f_{p}(\theta)}{\omega_{p}(\theta)} p_{K, p}(\theta)-\frac{f_{p}(\theta+\pi / \nu)}{\omega_{p}(\theta+\pi / \nu)} p_{K, p}(\theta+\pi / \nu)\right| d \theta, \\
K=0,1, \cdots, \lambda-1, p=1, \cdots, \lambda,
\end{array}
$$

each satisfy an inequality similar to the one in the statement of the theorem.

We have

$$
\begin{aligned}
I_{K, p} \leqq & \int_{0}^{2 \pi}\left|\frac{p_{K, p}(\theta)}{\omega_{p}(\theta)}\right|\left|f_{p}(\theta)-f_{p}(\theta+\pi / \nu)\right| d \theta \\
& +\int_{0}^{2 \pi}\left|f_{p}(\theta+\pi / \nu)\right|\left|\frac{p_{K, p}(\theta)}{\omega_{p}(\theta)}-\frac{p_{K, p}(\theta+\pi / \nu)}{\omega_{p}(\theta+\pi / \nu)}\right| d \theta \\
= & J_{1}+J_{2} .
\end{aligned}
$$

It is obvious that $J_{1} \leqq M_{1} C(\pi / \nu)$. We shall have shown that $J_{2}=O\left[(\pi / \nu)^{1 / \bar{m}}\right]$ if we establish that

$$
\begin{aligned}
\int_{0}^{2 \pi}\left|\frac{p_{K, p}(\theta)}{\omega_{p}(\theta)}-\frac{p_{K, p}(\theta+t)}{\omega_{p}(\theta+t)}\right| d \theta=O\left(|t|^{1 / \bar{m}}\right), \\
K=0,1, \cdots, \lambda-1, p=1, \cdots, \lambda ;
\end{aligned}
$$

and the remainder of our proof will be concerned with this relation.

Assuming that $0 \leqq t \leqq \delta_{j} / 2, j=1, \cdots, s$, we write

$$
\begin{aligned}
\int_{0}^{2 \pi} \leqq & \sum_{j=1}^{8}\left(\int_{\xi_{j}-\delta_{j}}^{\xi_{j}-t}+\int_{\xi_{j}}^{\xi_{j}+\delta_{j}-t}\right)+\sum_{j=1}^{8}\left(\int_{\xi_{j}-t}^{\xi_{j}}+\int_{\xi_{j}+\delta_{j}-t}^{\xi_{j}+\delta_{j}}\right) \\
& +\sum^{\prime} \int_{\xi_{j}+\delta_{j}}^{\xi_{j+1}-\delta_{j+1}}=\sum_{j=1}^{s} \sigma_{j}+\Sigma_{2}+\Sigma_{3},
\end{aligned}
$$


where $\xi_{0}+\delta_{0}=0, \xi_{8+1}-\delta_{8+1}=2 \pi$, and where in $\sum^{\prime}$ the index $j$ assumes all values from zero to $s$ for which $\xi_{j}<\xi_{j+1}$. The function $p_{K, p}(\theta) / \omega_{p}(\theta)$ is an analytic function of $\theta$ for $\xi_{j}+\delta_{j} \leqq \theta \leqq \xi_{j+1}-\delta_{j+1}+\frac{1}{2} \delta_{j+1}, j=0,1, \cdots, s-1, \xi_{j}<\xi_{j+1}$, and for $\xi_{1}+\delta_{s} \leqq \theta \leqq 2 \pi+\delta_{1}$; whence $\Sigma_{3}=O(t)$. By Lemma 6.1, $\Sigma_{2}=O\left(t^{1 / \bar{m}}\right)$. If $\omega_{p}\left(\xi_{j}\right) \neq 0$, then $\sigma_{j}=O(t)$. If on the contrary, $\zeta_{p}\left(\xi_{j}\right)=\beta_{j}$, then it is rather easily shown by using (6.5) and the identity $A B-C D=A(B-D)+D(A-C)$ that

$$
\begin{aligned}
\sigma_{j} \leqq\left. M\left(\int_{\xi_{j}-\delta_{j}}^{\xi_{j}-t}+\int_{\xi_{j}}^{\xi_{j}+\delta_{j}-t}\right)|| \frac{1}{2 \sin \frac{1}{2}\left(\theta-\xi_{j}\right)}\right|^{\left(m_{j}-1\right) / m_{j}} \\
-\left|\frac{1}{2 \sin \frac{1}{2}\left(\theta+t-\xi_{j}\right)}\right|^{\left(m_{j}-1\right) / m_{j}} \mid d \theta+O\left(t^{-1 / m_{j}}\right)=O\left(t^{-1 / \bar{m}}\right),
\end{aligned}
$$

which establishes (8.1) for $t \geqq 0$. The proof of the theorem is now complete.

We note in passing that (8.1) may easily be established for negative values of $t$ by a slight modification of the above argument.

In certain senses, the estimate of the coefficients given by Theorem 8.3 cannot be improved. For example, the function $g_{2}\left(z ; \frac{1}{2}\right)$ (for which the lemniscate of convergence has the index 2) has the property that $C(\delta) \equiv 0$, but the coefficients of its Jacobi series are $O\left(n^{-1 / 2}\right)$, but not $o\left(n^{-1 / 2}\right)$. Again, by using certain examples $\left({ }^{22}\right)$ in the theory of trigonometric series, it is easy to construct functions of class $H_{\Gamma}$, with $\bar{m}=1$, for which $C(\delta) \leqq M \delta \eta, 0<\eta<1$, and for which the quantities $\mu^{\nu}\left|a_{\lambda \nu+K}\right|$ are $O\left(n^{-\eta}\right)$ but not $o\left(n^{-\eta}\right)$.

THEOREM 8.4. If $F(z)$ is of bounded variation on $\Gamma$, then

(a) $\mu^{\nu} a_{\lambda \nu+K}=O\left(\nu^{-1 / \bar{m}}\right), \bar{m}>1$,

(b) $\mu^{\nu} a_{\lambda \nu+K}=o\left(\nu^{-1}\right), \bar{m}=1$.

It follows at once from the continuous, one-to-one nature of the correspondence between the boundaries of the regions $B$ and $B_{p}$, that under the present hypothesis on $F(z)$, the functions $f_{p}(\theta)$ are of bounded variation on any finite interval, as are also the functions $\omega_{p}(\theta)$ and $p_{K, p}(\theta)$. If $\bar{m}=1$, it is a consequence of the formulas in Theorem $7.6(\mathrm{~d})$ that the functions $\phi_{K}(\theta)$ are of bounded variation. Therefore since they are the boundary values of functions of class $H$, they are absolutely continuous $[15 ; 22$, p. 158], and their Fourier coefficients are $o\left(n^{-1}\right)[22$, p. 18]. This proves part (b) of the theorem.

Turning to part (a), we write

$$
\begin{aligned}
\int_{0}^{2 \pi} \phi_{K}(\theta) e^{-i \nu \theta} d \theta & =\sum^{\prime} \int_{\xi_{j}-\delta_{j}}^{\xi_{j}+\delta_{j}} \phi_{K}(\theta) e^{-i \nu \theta} d \theta+\sum^{\prime} \int_{\xi_{j}+\delta_{j}}^{\xi_{j+1}-\delta_{j+1}} \phi_{K}(\theta) e^{-i \nu \theta} d \theta, \\
& =\Sigma_{1}+\Sigma_{2},
\end{aligned}
$$

where $\xi_{0} \pm \delta_{0}=0, \quad \xi_{s+1}-\delta_{s+1}=2 \pi$, and $\sum^{\prime}$ has the same significance as in

(22) See for instance [22, p. 38, ex. 3] (the conjugate is $\in$ lip $\alpha$, by the theorem of Privaloff $[22$, p. 156]). 
(8.2). The function $\phi_{K}(\theta)$ is of bounded variation, $\xi_{j}+\delta_{j} \leqq \theta \leqq \xi_{j+1}-\delta_{j+1}$, $j=0,1, \cdots, s, \xi_{j}<\xi_{j+1}$, so by a classical theorem $\left[22\right.$, p. 18], $\Sigma_{2}=O\left(\nu^{-1}\right)$. If $\omega_{p}\left(\xi_{j}\right) \neq 0$, then $f_{p}(\theta) p_{K, p}(\theta) / \omega_{p}(\theta)$ is of bounded variation for $\theta$ in the closed interval $I:\left[\xi_{j}-\delta_{j}, \xi_{j}+\delta_{j}\right]$, and $\int_{I}\left[f_{p}(\theta) p_{K, p}(\theta) e^{-i \nu \theta} / \omega_{p}(\theta)\right] d \theta=O\left(\nu^{-1}\right)$, by the classical theorem. Suppose now that $\zeta_{p_{1}}(\xi)=\zeta_{p_{2}}(\xi)=\cdots=\zeta_{p_{m}}(\xi)=\beta$, where $\xi=\xi_{j}$. Then by referring to (6.5) we may write, for $\theta \in I$,

$$
\begin{aligned}
& \sum_{h=1}^{m} \frac{f_{p_{h}}(\theta) p_{K, p_{h}}(\theta)}{\omega_{p_{h}}(\theta)}=\sum_{h=1}^{m} \frac{f_{p_{h}}(\theta) \chi_{K, p_{h}}(\theta)}{\left(\zeta_{p_{h}}(\theta)-\beta\right)^{m-1}} \\
&=\frac{1}{|\theta-\xi|^{(m-1) / m}}\left[\frac{e^{-i[(m-1) / m] \psi(\theta)}|\theta-\xi|^{(m-1) / m}}{\left|2 \sin \frac{1}{2}(\theta-\xi)\right|^{(m-1) / m}}\right. \\
&\left.\cdot \sum_{h=1}^{m} \frac{f_{p_{h}}(\theta) \chi_{K, p_{h}}(\theta) e^{2 \pi i h / m}}{\left[A\left(W_{h}\right)\right]^{m-1}}\right],
\end{aligned}
$$

where $\chi_{K, p_{h}}(\theta)$ is a rational function of $\zeta_{p_{h}}(\theta)$ with no poles for $\theta \in I$. Since $\chi_{K, p_{h}}(\theta) /\left[A\left(W_{h}\right)\right]^{m-1}$ is an absolutely continuous function of $\theta$ for $\theta \in I$, and $\psi(\theta)$ is a step function, and $|\theta-\xi| /\left|\sin \frac{1}{2}(\theta-\xi)\right|$ is an analytic function of $\theta$ for $\theta \in I$, it follows that the quantity in brackets in (8.3) is of bounded variation for $\theta \in I$. Let us denote this quantity by $X(\theta)$. Another classical theorem $[3$, p. 494] now states that

$$
\lim _{n \rightarrow \infty} \nu^{-1 / m} \int_{I} X(\theta) \frac{e^{-i \nu \theta}}{|\theta-\xi|^{(m-1) / m}} d \theta=K_{1} X(\xi+0)+K_{2} X(\xi-0),
$$

where $K_{1}$ and $K_{2}$ are complex constants depending on $m$. The proof of part (a) is complete.

We observe that

$$
X(\xi \pm 0)=e^{-i[(m-1) / m] \psi(\xi \pm 0)} \chi_{K, p_{1}}(\xi) \cdot c_{1}^{1-m} \sum_{h=1}^{m} f_{p_{h}}(\xi \pm 0) e^{2 \pi i h / m}
$$

If $F(z)$ is of bounded variation on $\Gamma$, it can have only simple discontinuities on $\Gamma$. It can be shown that such discontinuities cannot occur on the curves $C_{k}$, which means that we can replace $f_{p_{h}}(\xi \pm 0)$ by $f_{p_{h}}(\xi)$ in (8.4). If we further suppose that $f_{p_{1}}(\xi)=f_{p_{2}}(\xi)=\cdots=f_{p_{m}}(\xi)$, then obviously $X(\xi \pm 0)=0$. Thus we have the following theorem:

THEOREM 8.5. If $F(z)$ is single-valued and of bounded variation on $\Gamma$, then $\mu^{\nu} a_{\lambda \nu+K}=o\left(\nu^{-1 / \bar{m}}\right), \bar{m} \geqq 1$.

The function $g_{2}\left(z ; \frac{1}{2}\right)$, which is absolutely continuous on each of the two contours of its lemniscate of convergence, has coefficients which are $O\left(n^{-1 / \bar{m}}\right)$, but not $o\left(n^{-1 / \bar{m}}\right)$; so the estimate in Theorem 8.4(a) cannot be improved. We cannot replace $o\left(\nu^{-1 / \bar{m}}\right)$ in Theorem 8.5 by $O\left(\nu^{-(1 / m)-\epsilon}\right)$, where $\epsilon$ is any fixed 
positive number, because the function $g_{2}(z ; q), q<\frac{1}{2}$, is absolutely continuous on its entire lemniscate of convergence (for which $\bar{m}=2$ ), but its Jacobi coefficients are $O\left(n^{q-1}\right)$ but not $o\left(n^{q-1}\right)$.

We conclude this section with two results analogous respectively to Parseval's theorem and to the Riesz-Fisher theorem.

Let $Q_{p}$ denote the series $\sum_{\nu=0}^{\infty} \sum_{K=0}^{\lambda-1}\left|\mu^{\nu} a_{\lambda \nu+K}\right|^{p}$.

THEOREM 8.6. If $F(z) \in H_{\Gamma}^{\alpha}, 1<\alpha \leqq 2$, then $Q_{\beta}$ is convergent, where

(a) $\beta=\alpha /(\alpha-1)$ for $\bar{m}=1$,

(b) $\beta>\alpha \bar{m} /(\alpha-1)$ for $\bar{m}>1$.

The theorem is an immediate consequence of Theorem 7.2, parts (a) and (c), and of the Hausdorff-Young theorem [22, p. 190]. The class $H^{2}$ is of particular interest because in this case $\beta=\alpha$; but the class $H_{\mathrm{r}}^{2}$ is of comparable interest only when $\bar{m}=1$. The example $g\left(z ; \frac{1}{2}\right)$ is worth mentioning in this connection. This function is of class $H_{\Gamma}^{\infty}$, and the $n$th partial sum of the corresponding series $Q_{2}$ is twice the Landau upper bound for the modulus of the $n$th partial sum of the Taylor series for an arbitrary function of class $H^{\infty}$, and is asymptotic to $(2 \log n) / \pi$.

THEOREM 8.7. Let $a_{0}, a_{1}, \cdots$ be any sequence of numbers such that for some $\mu>0, \sum_{\nu=0}^{\infty} \sum_{K=0}^{\lambda-1}\left|\mu^{\nu} a_{\lambda v+K}\right|^{\alpha}$ is finite, $1<\alpha \leqq 2$. There exists a function $F(z)$ regular and single-valued for $|\omega(z)|<\mu$ such that $\int \Gamma_{\rho}|F(z)| \beta\left|\omega^{\prime}(z)\right||d z|<M$ for $0<\rho<1$, where $\beta=\alpha /(\alpha-1)$, and for which the numbers $a_{n}$ are the coefficients of the Jacobi series with respect to the points $\alpha_{j}$.

It is easily established by means of the Hausdorff-Young theorem [33, p. 190], and with the aid of the theory of Abel means [33, p. 87], that functions $\Phi_{K}(w) \in H^{\beta}$ exist for which the numbers $\mu^{\nu} a_{\lambda \nu+K}$ are the Maclaurin coefficients; and the remainder of the proof may be supplied by reference to Theorem 7.4 and the remark at the end of $\$ 2$.

9. Convergence theorems obtained by using the functions $\phi_{K}(\theta)$. The work of $\S \S 4-7$ allows us to answer many questions concerning the convergence and summability of the Jacobi series by merely referring directly to the theory of Fourier series. We shall of course not attempt to give a catalogue of such convergence theorems here, but shall refer briefly to certain results which can perhaps be considered typical.

The first of these is an analogue of the Fejér-Lebesgue-Hardy theorem $[22$, p. 49].

ThEOREM 9.1. $\operatorname{Lim}_{n \rightarrow \infty} S_{n}^{(r)}(z)=F(z), r>0$, almost everywhere on $\Gamma$.

The theorems of $\S 7$ and the Fejer-Lebesgue-Hardy theorem establish this result immediately for the functions $\Psi_{K}(z)$ and their Jacobi series; and the proof may be completed by using a theorem of the author on the Cesàro method of summation [4, pp. 707-708], and Theorem 7.6(c). 
We turn next to convergence in the mean. It is well known that if $\left|\phi_{K}(\theta)\right|^{p}, p \geqq 1$, is integrable for $0 \leqq \theta \leqq 2 \pi$, then the Fourier series for $\phi_{K}(\theta)$ converges in the mean to $\phi_{K}(\theta)$ with index $p^{\prime}$, where $p^{\prime}=p$ if $p>1,0<p^{\prime}<1$ if $p=1[22$, p. 153]. The proof of the following theorem is easily supplied by using the results of $\S 7$ and the Minkowski inequality [18, p. 384].

THEOREM 9.2. If $F(z) \in H_{\Gamma}^{\alpha}, \alpha \geqq 1$, then

(a) $\lim _{n \rightarrow \infty} \int_{\Gamma}\left|\Psi_{K}(z)-\sum_{\nu=0}^{n} a_{\lambda \nu+K}[\omega(z)]^{\nu}\right|^{\alpha^{\prime}}\left|\omega^{\prime}(z)\right||d z|=0, K=0,1, \cdots, \lambda-1$,

$$
\lim _{n \rightarrow \infty} \int_{\Gamma}\left|F(z)-S_{n}(z)\right|^{\prime}\left|\omega^{\prime}(z)\right||d z|=0
$$

The number $\alpha^{\prime}$ is described by the formulas in Theorem 7.2 if $\alpha>1$. If $\alpha=1$, then $0<\alpha^{\prime}<1$.

Uniform convergence theorems may be obtained by imposing suitable conditions directly on the functions $\phi_{K}(\theta)$. We have, for instance, as an analogue of Dirichlet's theorem [22, p. 25] the following result:

THEOREM 9.3. If the functions $\phi_{K}(\theta), K=0,1, \cdots, \lambda-1$, are of bounded variation for $0 \leqq \theta \leqq 2 \pi$, then $\lim _{n \rightarrow \infty} S_{n}(z)=F(z)$ uniformly for $z$ in $\bar{D}$.

Obviously there is a theorem of this type for each of the many tests for convergence of the Fourier series. But it would seem to be of interest to derive such results from conditions imposed directly on the function $F(z)$ rather - than on the functions $\phi_{K}(\theta)$, particularly if $\bar{m}>1$. The functions $\Psi_{K}(z)$ may behave much less "smoothly" than $F(z)$ in the neighborhood of a multiple point on $\Gamma$, and the convergence properties of the series $\sum_{0}^{\infty} a_{\lambda_{\nu+K}}[\omega(z)]^{v}$ may not adequately reflect those of the Jacobi series for the function $F(z)$. For example, the function $g_{2}(z, q), 0<q<\frac{1}{2}$, is absolutely continuous on its lemniscate of convergence, and its Jacobi series converges uniformly on this lemniscate $\left({ }^{23}\right)$; but the corresponding functions $\Psi_{0}(z)$ and $\Psi_{1}(z)$ are both infinite for $z=0$ and their Jacobi series are properly divergent at this point.

In the remainder of this paper we shall introduce methods which enable us to derive convergence theorems without reference to the properties of the functions $\Psi_{K}(z)$ and their series.

10. The function $H(Z, w)$ and the integral formulas for $S_{\lambda_{n+\lambda-1}}(z ; F)$. Let $F(z)$ be any analytic function, not necessarily of class $H_{\Gamma}$, for which $\Gamma$ is the lemniscate of convergence. We define the function $H(Z, w ; F)$, or $H(Z, w)$, for any $Z$ and for $|w|<1$, as follows:

$(10.1) H(Z, w)=\Phi_{0}(w)+\left(Z-\alpha_{1}\right) \Phi_{1}(w)+\cdots+\left(Z-\alpha_{1}\right) \cdots\left(Z-\alpha_{\lambda-1}\right) \Phi_{\lambda-1}(w)$.

(23) See Theorem 11.7. 
The variables $Z$ and $w$ are to be considered as independent here. For a fixed $w$, this function is a polynomial in $Z$ which coincides with $F(Z)$ in the points $Z=z_{p}(w), p=1, \cdots, \lambda$. Therefore for $w \neq b_{j}, j=1, \cdots, \lambda_{1}$, we may use the Lagrange interpolation formula $[20$, p. 50$]$ to write $\left({ }^{24}\right)$

$$
H(Z, w)=\sum_{p=1}^{\lambda} \frac{F\left(z_{p}(w)\right)}{\omega^{\prime}\left(z_{p}(w)\right)} \frac{\omega(Z)-w}{Z-z_{p}(w)}, \quad|w|<1 .
$$

The quotients in this formula are of course supposed to be defined by their limiting values for $Z=z_{p}(w), p=1, \cdots, \lambda$. If $F(z) \equiv 1$, then $H(Z, w) \equiv 1$, so for any number $f$ we have

$$
\begin{aligned}
H(Z, w)-f=\sum_{p=1}^{\lambda} \frac{F\left(z_{p}(w)\right)-f}{\omega^{\prime}\left(z_{p}(w)\right)} \frac{\omega(Z)-\mu w}{Z-z_{p}(w)}, \\
|w|<1, w \neq b_{j}, j=1, \cdots, \lambda_{1} .
\end{aligned}
$$

THEOREM 10.1. If $F(z) \in H_{\Gamma}^{\alpha}$, then $H(Z, w)$, considered as a function of $w$, belongs to the class $H^{\alpha^{\prime}}$, where $\alpha^{\prime}$ is given by the formulas of Theorem 7.2.

The result is an immediate consequence of (10.1) and Theorem 7.2.

THEOREM 10.2. If $F(z) \in H_{\Gamma}^{\alpha}$, there exists a finite-valued function $H^{*}(Z, w)$ defined for each value of $Z$ and for almost every $w,|w|=1$, by the equation

$H^{*}(Z, w)=\Phi_{0}(w)+\left(Z-\alpha_{1}\right) \Phi_{1}(w)+\cdots+\left(Z-\alpha_{1}\right) \cdots\left(Z-\alpha_{\lambda-1}\right) \Phi_{\lambda_{-1}}(w)$, and such that

(a) $H(Z, w) \rightarrow H^{*}(Z, W)$ and $H\left(z_{p}(w), w\right) \rightarrow H^{*}\left(z_{p}(W), W\right), p=1, \cdots, \lambda$, for almost every point $W$ for which $|W|=1$ as $w \rightarrow W$ along any $S$-path of the regions $|w|<1$;

$$
H^{*}(Z, w)=\sum_{p=1}^{\lambda} \frac{F\left(z_{p}(w)\right)}{\omega^{\prime}\left(z_{p}(w)\right)} \frac{\omega(Z)-\mu w}{Z-z_{p}(w)}
$$

for almost every wor which $|w|=1$;

$$
\begin{aligned}
& \int_{\gamma}|H(Z, w)| \alpha^{\prime}|d w|<\infty, \quad \int_{\gamma}\left|H\left(z_{p}(w), w\right)\right| \alpha|d w|<\infty, \\
& p=1, \cdots, \lambda,
\end{aligned}
$$

where $\alpha^{\prime}$ is given by the formulas in Theorem 7.2.

The theorem is easily proved by using (10.1), (10.2), and Theorem 7.6. We shall henceforth use the single symbol $H(Z, w)$ to designate the complete function consisting of $H(Z, w),|w|<1$, and $H^{*}(Z, w),|w|=1$. Let $h_{p}\left(\theta^{\prime}, \theta\right)$

(24) The function $H(Z, w)$ is independent of the order of the points $\alpha_{j}$. Thus in studying this function, we are turning to Jacobi's original point of view. 
$=H\left(z_{p}\left(e^{i \theta^{\prime}}\right), e^{i \theta}\right)$ and let

$$
\chi_{p, q}\left(\theta^{\prime}, \theta\right)=\frac{1}{\omega_{q}(\theta)} \frac{\mu e^{i \theta^{\prime}}-\mu e^{i \theta}}{\zeta_{p}\left(\theta^{\prime}\right)-\zeta_{q}(\theta)}=\frac{\prod_{j=1, j \neq p}^{\lambda}\left(\zeta_{q}(\theta)-\zeta_{j}\left(\theta^{\prime}\right)\right)}{\lambda \prod_{j=1}^{\lambda_{1}}\left(\zeta_{q}(\theta)-\beta_{j}\right)^{m_{j}-1}} .
$$

Then

$$
\begin{gathered}
h_{p}\left(\theta^{\prime}, \theta\right)=\sum_{q=1}^{\lambda} f_{q}(\theta) \chi_{p, q}\left(\theta^{\prime}, \theta\right), \\
h_{p}\left(\theta^{\prime}, \theta\right)-f=\sum_{q=1}^{\lambda}\left(f_{q}(\theta)-f\right) \chi_{p, q}\left(\theta^{\prime}, \theta\right) .
\end{gathered}
$$

For a fixed value of $\theta^{\prime}$, formulas (10.5) and (10.6) are true for almost every value of $\theta$; and if it should happen for a given value of $\theta$ that they are true for some value of $\theta^{\prime}$, then they are true for all other values of $\theta^{\prime}$.

The Jacobi series for $H(Z, \omega(z) / \mu)$ with respect to the points $\alpha_{j}$ is the series

$$
\begin{aligned}
\sum_{\nu=0}^{\infty}\left\{a_{\lambda \nu}+a_{\lambda \nu+1}\left(Z-\alpha_{1}\right)\right. & +\cdots \\
& \left.+a_{\lambda \nu+\lambda-1}\left(Z-\alpha_{1}\right) \cdots\left(Z-\alpha_{\lambda-1}\right)\right\}[\omega(z)]^{\nu} .
\end{aligned}
$$

The Taylor series for $H(Z, w)$ about the point $w=0$ is obtained from (10.7) by substituting $\mu w$ for $\omega(z)$.

THEOREM 10.3. If $F(z) \in H_{\Gamma}$, the series

$$
\sum_{\nu=0}^{\infty}\left\{a_{\lambda \nu}+a_{\lambda \nu+1}\left(Z-\alpha_{1}\right)+\cdots+a_{\lambda \nu+\lambda-1}\left(Z-\alpha_{1}\right) \cdots\left(Z-\alpha_{\lambda-1}\right)\right\} \mu^{\nu} e^{i \nu \theta}
$$

is the Fourier series for the function $H\left(Z, e^{i \theta}\right)$, considered as a function of $\theta$.

The theorem is a consequence of (10.7), Theorem 10.1 and the theorem of $F$. and M. Riesz to which allusion has previously been made [15, p. 42].

Henceforth we assume invariably that $F(z) \in H_{\Gamma}$.

We write:

$$
\begin{gathered}
S_{n}\left(z_{p}\left(e^{i \theta}\right)\right)=s_{p, n}(\theta), \\
\sum_{\nu=0}^{n}\left\{a_{\lambda \nu}+a_{\lambda \nu+1}\left(\zeta_{p}\left(\theta^{\prime}\right)-\alpha_{1}\right)+\cdots\right. \\
\left.+a_{\lambda \nu+\lambda-1}\left(\zeta_{p}\left(\theta^{\prime}\right)-\alpha_{1}\right) \cdots\left(\zeta_{p}\left(\theta^{\prime}\right)-\alpha_{\lambda-1}\right)\right\} \mu^{\nu} e^{i \nu \theta} \\
=\sum_{\nu=0}^{n} c_{p, \nu}\left(\theta^{\prime}\right) e^{i \nu \theta}=\sigma_{p, n}\left(\theta^{\prime}, \theta\right) ; \\
\sigma_{p, n}(\theta, \theta)=\sigma_{p, n}(\theta)=s_{p, \lambda n+\lambda-1}(\theta) .
\end{gathered}
$$

Dirichlet's integral formulas [22, pp. 20-21] for $\sigma_{p, n}\left(\theta^{\prime}, \theta\right)$ take the following forms: 


$$
\begin{gathered}
\sigma_{p, n}\left(\theta^{\prime}, \theta\right)=\frac{1}{\pi} \int_{-\pi}^{\pi} h_{p}\left(\theta^{\prime}, \theta+\tau\right) K_{n}(\tau) d \tau \\
\sigma_{p, n}\left(\theta^{\prime}, \theta\right)-f=\frac{1}{\pi} \int_{0}^{\pi}\left[h_{p}\left(\theta^{\prime}, \theta+\tau\right)+h_{p}\left(\theta^{\prime}, \theta-\tau\right)-2 f\right] K_{n}(\tau) d \tau
\end{gathered}
$$

both formulas holding for all $\theta$ and $\theta^{\prime}$. When $\theta^{\prime}=\theta$, these formulas become useful expressions for the $\lambda(n+1)$ th partial sum of the series (1.1). We obtain the following result by referring to Theorem 8.2 :

THEOREM 10.4. If $\lim _{n \rightarrow \infty} \sigma_{p, n}(\theta)=f(\theta)$ uniformly on a set $E$, then $\lim _{n \rightarrow \infty}$ $s_{p, n}(\theta)=f(\theta)$ uniformly on $E$.

11. Convergence tests. When $\bar{m}=1$, the integrals (10.8) and (10.9) are essentially no more complicated than the ordinary Dirichlet integral in the theory of Fourier series. However, when $\bar{m}>1$ the integrals must be studied by methods which are modifications or extensions of certain of the methods of Fourier analysis. Some of the extensions are trivial, some non-trivial and of interest in themselves.

The methods are perhaps best illustrated by deriving convergence criteria for the Jacobi series analogous to the de la Vallée Poussin test and to some of the tests which it includes. Accordingly, we shall devote this final section to such derivations.

It will be convenient to have formal statements of a few simple results on functions of bounded variation. If $\phi(t)$ is a complex-valued function of the real variable $t$ which is of bounded variation on the interval $[0, t]$, we shall denote the absolute (or total) variation $\left[16\right.$, p. 96] $\left({ }^{25}\right)$ of $\phi(t)$ over the interval $[0, t]$ by $V(\phi ; t)$. Obviously $V(R(\phi) ; t) \leqq V(\phi ; t)$ and $V(J(\phi) ; t) \leqq V(\phi ; t)$.

LEMMA 11.1. Let $f(t, \theta)$ be a real function of the real variables $t$ and $\theta$, and let $f(0, \theta)=0$. A necessary and sufficient condition that $f(t, \theta)$ be of bounded variation in $t$ for $0 \leqq t \leqq T$ and for each $\theta$ on a set of $E$, and that $\lim _{t \rightarrow 0} f(t, \theta)=\lim _{t \rightarrow 0} V(f ; t)$ $=0$ uniformly for $\theta \in E$, is that $f(t, \theta)$ may be expressed as the difference of two non-negative functions of $t$ and $\theta$ which are monotone non-decreasing functions of $t$ for any fixed values of $\theta \in E$, and which approach zero uniformly, $\theta \in E$, as $t \rightarrow 0$.

The sufficiency is obvious, and the necessity may be proved by simply writing down the usual expression for $f(t, \theta)$ in terms of its upper and lower variations with respect to $t[16$, p. $98 ; 18$, p. 356].

LEMMA 11.2. Let $f(t, \theta)$ and $g(t, \theta)$ be complex functions of the real variables $t$ and $\theta$, defined and integrable with respect to $t$ on the closed interval $[0, T]$ for each $\theta$ on a set $E$. If $g(t, \theta)$ and the function

$\left.{ }^{25}\right)$ We shall use Saks' terminology in referring to functions of bounded variation. 


$$
F(t, \theta)=\left\{\begin{array}{l}
\frac{1}{t} \int_{0}^{t} f(\tau, \theta) d \tau, \quad t \neq 0 \\
f(0, \theta), \quad t=0
\end{array}\right.
$$

are of bounded variation in $t$ over the interval $[0, T]$ for each $\theta \in E$, then so is the function

$$
G_{1}(t, \theta)=\left\{\begin{array}{l}
\frac{1}{t} \int_{0}^{t} g(\tau, \theta) f(\tau, \theta) d \tau, \quad t \neq 0, \\
g(+0, \theta) f(0, \theta), \quad t=0 .
\end{array}\right.
$$

Moreover if

$$
|F(t, \theta)|+V(F ; t) \leqq M(F), \quad|g(t, \theta)|+V(g ; t) \leqq M(g),
$$

for $t$ on the interval $[0, T], \theta \in E$, then

$$
|G(t, \theta)| \leqq A M(F) M(g), \quad V(G ; t) \leqq A M(F) M(g),
$$

for $t$ on the interval $[0, T], \theta \in E$, where $A$ is an absolute constant. In particular, if $\lim _{t \rightarrow 0} F(t, \theta)=\lim _{t \rightarrow 0} V(F ; t)=0$, or if $\lim _{t \rightarrow 0} g(t, \theta)=\lim _{t \rightarrow 0} V(g ; t)=0$ uniformly, $\theta \in E$, then $\lim _{t \rightarrow 0} G(t, \theta)=\lim _{t \rightarrow 0} V(G ; t)=0$ uniformly for $\theta \in E$.

It is sufficient to prove the theorem for the case in which $T>0$ and $F(t, \theta)$ and $g(t, \theta)$ are real, non-negative, non-decreasing functions of $t$ for each $\theta \in E$. We write

$$
G(t, \theta)=\frac{1}{t} \int_{0}^{t} g F d \tau+\frac{1}{t} \int_{0}^{t} g \frac{\partial F}{\partial \tau} \tau d \tau=I_{1}+I_{2}
$$

A well known theorem $\left[17\right.$, p. 100] enables us to assert immediately $I_{1}$ is nondecreasing on the interval $[0, T]$; and clearly $I_{1} \leqq M(F) M(g)$. We integrate by parts in $I_{2}$ :

$$
I_{2}=\int_{0}^{t} g \frac{\partial F}{\partial \tau} d \tau-\frac{1}{t} \int_{0}^{t}\left[\int_{0}^{u} g \frac{\partial F}{\partial \tau} d \tau\right] d u
$$

Each of these expressions is a non-decreasing function of $t$, and $\int_{0}^{t} g[\partial F / \partial \tau] d \tau$ $\leqq M(g)[F(t, \theta)-F(0, \theta)] \leqq M(g) M(F)$. We leave the remaining details to the reader.

Lemma 11.3. Let $f(t, \theta)$ be defined as in Lemma 11.2. If $\lim _{t \rightarrow 0} \int_{0}^{t}|f(\tau, \theta)| d \tau$ $=0$ uniformly, $\theta \in E$, then the function

$$
\phi(t, \theta)=\left\{\begin{array}{l}
\frac{1}{t} \int_{0}^{t} \tau f(\tau, \theta) d \tau, \quad t \neq 0, \\
0, \quad t=0,
\end{array}\right.
$$


is of bounded variation in $t$ over the interval $[0, T]$ for each $\theta \in E$, and

$$
\lim _{t \rightarrow 0} \phi(t, \theta)=\lim _{t \rightarrow 0} \frac{1}{t} \int_{0}^{t} \tau|f(\tau, \theta)| d \tau=\lim _{t \rightarrow 0} V(\phi ; t)=0
$$

uniformly, $\theta \in E$.

A proof may be given by the methods used in the proof of the preceding lemma; we again leave the details to the reader.

We now let $f(\theta)$ be an arbitrary complex single-valued function of $\theta$, and we write

$$
\begin{aligned}
& H_{p}(\theta, \tau ; f(\theta))=h_{p}(\theta, \theta+\tau)+h_{p}(\theta, \theta-\tau)-2 f(\theta), \\
& J_{p}(t, \theta ; f(\theta))=J_{p}(t)=\left\{\begin{array}{l}
\frac{1}{t} \int_{0}^{t} H_{p}(\theta, \tau ; f(\theta)) d \tau, \quad t \neq 0, \\
0, \quad t=0 .
\end{array}\right.
\end{aligned}
$$

This equation defines a single-valued function of $t$ and $\theta$ for all values of $\theta$ for which $f(\theta)$ is defined, and for all values of $t$.

If a function $f(t, \theta)$, defined as in Lemma 11.2, is of bounded variation in $t, 0 \leqq t \leqq T, T>0$, for each $\theta$ on a set $E$, where $T$ is independent of $\theta$, and if $\lim _{t \rightarrow 0} f(t, \theta)=\lim _{t \rightarrow 0} V(f ; t)=0$ uniformly, $\theta \in E$, then we shall say that this function has property $A$ uniformly on $E$. If $E$ consists of a single point, we shall simply say that $f(t, \theta)$ has property $\mathcal{A}\left({ }^{26}\right)$.

The fundamental theorem of this section will now be stated:

THEOREM 11.1. (a) If for some number $f, J_{p}(t, \theta ; f)$ has property $\mathcal{A}$, then $\lim _{n \rightarrow \infty} s_{p, n}(\theta)=f$.

(b) If $f(\theta)$ is defined on a set $E$, if $|f(\theta)| \leqq M, \theta \in E$, and if $J_{p}(t, \theta ; f(\theta))$ has property $A$ uniformly on $E$, then $\lim _{n \rightarrow \infty} s_{p, n}(\theta)=f(\theta)$ uniformly on $E$.

To prove the theorem, we first establish a result of the Riemann-Lebesgue type.

Lemma 11.4. Assume that $\phi(t)$ is a bounded integrable function of $t$ for $a \leqq t \leqq b . T h e n$

$$
\lim _{n \rightarrow \infty} \int_{a}^{b} h_{p}\left(\theta^{\prime}, \theta+\tau\right) \phi(\tau)\left\{\begin{array}{l}
\sin n \tau \\
\cos n \tau
\end{array}\right\} d \tau=0, \quad p=1, \cdots, \lambda,
$$

uniformly for all values of $\theta$ and $\theta^{\prime}$.

For we have

(26) It is perhaps worth while to point out that if $f(t, \theta)$ is of bounded variation in $t, 0 \leqq t \leqq T$, $T>0$, and if $\lim _{t \rightarrow 0} f(t, \theta)=0$, then $\lim _{t \rightarrow 0} V(f ; t)=0$; but uniformity of the first limit in $\theta$ does not imply uniformity of the second limit in $\theta$. 


$$
\begin{aligned}
& \int_{a}^{b} h_{p}\left(\theta^{\prime}, \theta+\tau\right) \phi(\tau)\left\{\begin{array}{l}
\sin n \tau \\
\cos n \tau
\end{array}\right\} d \tau \\
& =\int_{a}^{b} \phi_{0}(\theta+\tau) \phi(\tau)\left\{\begin{array}{l}
\sin n \tau \\
\cos n \tau
\end{array}\right\} d \tau+\left(\zeta_{p}\left(\theta^{\prime}\right)-\alpha_{1}\right) \int_{a}^{b} \phi_{1}(\theta+\tau) \phi(\tau)\left\{\begin{array}{l}
\sin n \tau \\
\cos n \tau
\end{array}\right\} d \tau \\
& +\cdots+\left(\zeta_{p}\left(\theta^{\prime}\right)-\alpha_{1}\right) \cdots\left(\zeta_{p}\left(\theta^{\prime}\right)-\alpha_{\lambda-1}\right) \int_{a}^{b} \phi_{\lambda-1}(\theta+\tau) \phi(\tau)\left\{\begin{array}{l}
\sin n \tau \\
\cos n \tau
\end{array}\right\} d \tau,
\end{aligned}
$$

and we may apply a familiar extension of the Riemann-Lebesgue theorem $[22$, p. 22] to each of the terms in this sum, which are periodic functions of $\theta$ and $\theta^{\prime}$.

Thus for any fixed $\delta, 0<\delta<\pi$, we find that

$$
\lim _{n \rightarrow \infty} \int_{\delta}^{\pi} H_{p}(\theta, \tau ; f) K_{n}(\tau) d \tau=0
$$

uniformly, $\theta \in E$. We write

$$
\int_{0}^{\delta} H_{p}(\theta, \tau ; f) K_{n}(\tau) d \tau=\int_{0}^{\delta} J_{p}(\tau) K_{n}(\tau) d \tau+\int_{0}^{\delta} J_{p}^{\prime}(\tau) \tau K_{n}(\tau) d \tau .
$$

We apply Lemma 11.1 to the real and imaginary parts of $J_{p}(t)$; and by proceeding exactly as in the classical proof of Dirichlet's theorem [22, pp. 25-26; 18, p. 407 ], we can show that for an arbitrary positive number $\epsilon$, there exists a number $\delta^{\prime}$ independent of $n$ and $\theta$ such that if $\delta<\delta^{\prime}$

$$
\left|\int_{0}^{\delta} J_{p}(\tau) K_{n}(\tau) d \tau\right|<\epsilon, \quad \theta \in E, n=0,1,2, \cdots
$$

Suppose now that $j(t)$ is one of the monotone components of $J_{p}(t)$ referred to in Lemma 11.1. Then there exists a number $\delta^{\prime \prime}$ independent of $n$ and $\theta$ such that if $\delta<\delta^{\prime \prime}$ then

$$
\int_{0}^{\delta} j^{\prime}(\tau) \tau K_{n}(\tau) d \tau \leqq C \int_{0}^{\delta} j^{\prime}(\tau) d \tau \leqq C j(\delta)<\epsilon, \theta \in E, n=0,1, \cdots,
$$

where $C$ is an absolute constant. Inequalities similar to (11.3) may be established for each of the other monotone components of $J_{p}(t)$. The proof is completed by combining these inequalities with (11.1) and (11.2), and by applying Theorem 10.4.

The theorems in the remainder of this section contain sufficient conditions for convergence which are imposed directly upon the function $F(z)$ or upon its transform on the surface $R$.

The first of these theorems is an exact analogue of de la Vallee Poussin's test. 
THEOREM 11.2. If $\zeta_{p_{1}}(\xi)=\zeta_{p_{2}}(\xi)=\cdots=\zeta_{p_{n}}(\xi), m \geqq 1$, and if numbers $f_{h}$, $h=1, \cdots, m$, can be chosen so that the functions

$$
K_{p_{h}}(t)=\left\{\begin{array}{l}
\frac{1}{t} \int_{0}^{t}\left(f_{p_{h}}(\xi+\tau)+f_{p_{h}}(\xi-\tau)-2 f_{h}\right) d \tau, t \neq 0, \\
0, t=0,
\end{array} \quad h=1, \cdots, m,\right.
$$

all have property $\mathcal{A}$, then $\lim _{n \rightarrow \infty} s_{p_{1}, n}(\xi)=\bar{f}$, where $\bar{f}=\sum_{1}^{m} f_{h} / m$.

We give the proof by establishing the following lemma:

LEMma 11.5. If $K_{p_{h}}(t)$ has property $A, h=1, \cdots, m$, then $J_{p_{1}}(t, \xi ; \bar{f})$ has the property $A$.

We write

$$
\begin{aligned}
J_{p_{1}}(t, \xi ; \bar{f})= & \sum_{h=1}^{m} \frac{1}{t} \int_{0}^{t}\left[f_{p_{h}}(\xi+\tau)+f_{p_{h}}(\xi-\tau)-2 f_{h}\right] \chi_{p_{1}, p_{h}}(\xi, \xi+\tau) d \tau \\
+ & \sum_{h=1}^{m} \frac{1}{t} \int_{0}^{t} \frac{f_{p_{h}}(\xi-\tau)-f_{h}}{\omega_{p_{h}}(\xi-\tau)}\left[\frac{\chi_{p_{1}, p_{h}}(\xi, \xi-\tau)-\chi_{p_{1}, p_{h}}(\xi, \xi+\tau)}{\tau^{1 / m}}\right] \\
& \cdot \frac{\omega_{p_{h}}(\xi-\tau)}{\tau^{(m-1) / m} \tau d \tau} \\
& +\sum_{h=1}^{m} \frac{1}{t} \int_{0}^{t}\left(f_{h}-\bar{f}\right)\left[\chi_{p_{1}, p_{h}}(\xi, \xi+\tau)+\chi_{p, p_{h}}(\xi, \xi-\tau)\right] d \tau \\
& +\sum^{\prime} \frac{1}{t} \int_{0}^{t}\left[\left(f_{q}(\xi+\tau)-\bar{f}\right) \chi_{p_{1}, q}(\xi, \xi+\tau)\right. \\
= & \sum_{h=1}^{m} I_{h}^{(1)}+\sum_{h=1}^{m} I_{h}^{(2)}+\sum_{h=1}^{m} I_{h}^{(3)}+\sum_{q}^{\prime} I_{q}^{(4)}, \quad t \neq 0,
\end{aligned}
$$

where $\sum^{\prime}=\sum_{q=1}^{\lambda}, q \neq p_{1}, p_{2}, \cdots, p_{m}\left({ }^{27}\right)$. If $m>1$, then for some subscript $j$, we have $\xi \equiv \xi_{j}(\bmod 2 \pi), \zeta_{p_{1}}(\xi)=\beta_{j}$, and $m=m_{j}$; and

$$
\chi_{p_{1}, q}(\xi, \xi+\tau)=\frac{\Pi^{\prime}\left[\zeta_{q}(\xi+\tau)-\zeta_{n}(\xi)\right]}{\lambda \prod^{\prime \prime}\left[\zeta_{q}(\xi+\tau)-\beta_{n}\right]^{m_{n}-1}},
$$

where $\Pi^{\prime}=\prod_{n=1}^{\lambda}, n \neq p_{1}, p_{2}, \cdots, p_{m}$, and $\Pi^{\prime \prime}=\prod_{n=1}^{\lambda_{1}}, n \neq j$. Thus the function $\chi_{p_{1}, p_{h}}(\xi, \xi+\tau)$ is a rational function of $\zeta_{p_{h}}(\xi+\tau)$ with no poles for $-\delta_{j} \leqq \tau \leqq \delta_{j}$, and consequently is an absolutely continuous function of $\tau$ in this interval. Applying Lemma 11.2, we find that $I_{h}^{(1)}$ is of bounded variation in an interval to the right of $t=0$, and $\lim _{t \rightarrow 0} I_{h}^{(1)}=0$.

Now

${ }^{(27)}$ We may write $I_{p}^{(h)}=0$ for $t=0$. 
$\left|\chi_{p_{1}, p_{h}}(\xi, \xi-\tau)-\chi_{p_{1}, p_{h}}(\xi, \xi+\tau)\right| \leqq M\left|\zeta_{p_{h}}(\xi-\tau)-\zeta_{p_{h}}(\xi+\tau)\right| \leqq M_{1}|\tau|^{1 / m}$ for $|\tau| \leqq \delta_{j}$, according to (6.7); and, as we have seen in the proof of Lemma 6.1, $\left|\omega_{p_{h}}(\xi-\tau)\right| /|\tau|^{(m-2) / m}$ is uniformly bounded for $|\tau| \leqq \delta_{j}$. Therefore, since $\lim _{t \rightarrow 0} \int_{0}^{t}\left|\left(f_{p_{h}}(\xi-\tau)-f_{h}\right) / \omega_{p_{h}}(\xi-\tau)\right| d \tau=0$, we may apply Lemma 11.3 to $I_{h}^{(2)}$, and we find that it is of bounded variation for $|t| \leqq \delta_{j}$ and $\lim _{t \rightarrow 0} I_{h}^{(2)}=0$.

Next we notice that

$$
\lim _{t \rightarrow 0} \chi_{p_{1}, p_{h}}(\xi, \xi+t)=\lim _{z \rightarrow \beta_{j}} \frac{\omega(z)-\omega\left(\beta_{j}\right)}{\omega^{\prime}(z)\left(z-\beta_{j}\right)}=\frac{1}{m}, \quad h=1, \cdots, m,
$$

where $z=\zeta_{p_{h}}(\xi+t)$. Since $\sum_{h=1}^{m}\left(f_{h}-\bar{f}\right)=0$, we find from Lemma 11.2 that $I_{h}^{(3)}$ is of bounded variation for $|t| \leqq \delta_{j}$ and $\lim _{t \rightarrow 0} I_{h}^{(3)}=0$.

In connection with $I_{q}^{(4)}$, we shall state for future reference a result which is rather stronger than the one needed at present $\left({ }^{28}\right)$.

LEMMA 11.6. If $\phi(\theta)$ is a function such that $\phi(\theta) / \omega_{q}(\theta)$ is integrable on an interval $[a, b], a<b$, and if a number $T$ exists, $0<T \leqq(b-a) / 2$, such that $\left|\zeta_{p}(\theta)-\zeta_{q}(\theta+t)\right|$ is bounded from zero for all $\theta$ on the interval $[a+T, b-T]$, and for $-T \leqq t \leqq T$, then the function

$$
\Phi_{p, q}(t)=\Phi_{p, q}(t, \theta ; \phi)=\left\{\begin{array}{l}
\frac{1}{t} \int_{0}^{t} \phi(\theta+\tau) \chi_{p, q}(\theta, \theta+\tau) d \tau, \quad t \neq 0, \\
0, \quad t=0,
\end{array}\right.
$$

is of bounded variation in $t$ for $-T \leqq t \leqq T$ and for each $\theta$ in the interval $[a+T, b-T]$, and

$$
\begin{aligned}
\lim _{t \rightarrow 0} \Phi_{p, q}(t) & =\lim _{t \rightarrow 0} \frac{1}{t} \int_{0}^{t}\left|\phi(\theta+\tau) \chi_{p, q}(\theta, \theta+\tau)\right| d \tau \\
& =\lim _{t \rightarrow 0} V\left(\Phi_{p, q} ; t\right)=0
\end{aligned}
$$

uniformly for $\theta$ on the interval $[a+T, b-T]$.

The proof of this lemma may be given by referring to Lemma 11.3 , in which we let

$$
f(t, \theta)=\frac{\phi(\theta+t)}{\omega_{Q}(\theta+t)} \cdot \frac{1}{\zeta_{q}(\theta)-\zeta_{q}(\theta+t)} \cdot \frac{\mu e^{i \theta}-\mu e^{i(\theta+t)}}{t} .
$$

The application of Lemma 11.6 to $I_{q}^{(4)}$ is obvious. The proof of Lemma 11.5

(28) Lemmas 11.6 and 11.8 are stated in a form in which they are useful in obtaining certain results concerning the Cesàro means of the Jacobi series which the author intends to publish elsewhere. Thus the functions $Y_{p, q}(t, \theta)$ and $y(t, \theta)$ in Lemma 11.8 and its proof are not needed in the present discussion. 
for $m>1$ is completed by the observation that the sum of a finite number of functions which have property $\mathcal{A}$, itself has property $A$; we have shown $J_{p_{1}}(t, \xi ; \bar{f})$ to be such a sum.

The case $m=1$ is treated similarly, but the details are simpler, and will be omitted.

Using the notation of this theorem, we may state a test analogous to Dini's test:

THEOREM 11.3. If the functions

$$
\left|\frac{f_{p_{h}}(\xi+t)+f_{p_{h}}(\xi-t)-2 f_{h}}{t}\right|, \quad h=1, \cdots, m,
$$

are integrable in an interval to the right of $t=0$, then $\lim _{n \rightarrow \infty} s_{p_{1}, n}(\xi)=\bar{f}$.

A proof may be given by showing that the conditions in the hypothesis of Theorem 11.2 are satisfied; this may be conveniently done by using the method usually employed to show that de la Vallée Poussin's test includes Dini's test $[18$, p. $410 ; 22$, p. 36$]$. The theorem may also be proved by breaking up the integral in (10.9) into a number of integrals which correspond to the functions $I_{h}^{(1)}, I_{h}^{(2)}, I_{h}^{(3)}, I_{q}^{(4)}$ of the proof of Theorem 11.2 , and by showing that the Riemann-Lebesgue theorem applies to each one.

The following result can easily be established by using Theorem 11.3.

THEOREM 11.4. Let $\beta$ be a point of $\Gamma$ of multiplicity $m, m \geqq 1$, belonging to the $m$ curves $C_{k_{1}}, C_{k_{2}}, \cdots, C_{k_{m}}$. If $\left|F(z)-F_{h}\right| \leqq M|z-\beta| \eta, \eta>0$, for almost every $z \in C_{k_{h}}$ in some neighborhood of $\beta, h=1, \cdots, m$, then $\lim _{n \rightarrow \infty} S_{n}(\beta)=\sum_{1}^{m} F_{h} / m$.

Another consequence of Theorem 11.2 worth mentioning is the following theorem.

TheOREM 11.5. Let $\beta$ be a point of $\Gamma$ of multiplicity $m, m \geqq 1$, belonging to the $m$ curves $C_{k_{1}}, C_{k_{2}}, \cdots, C_{k_{m}}$. If $F(z)$ is of bounded variation on some arc of $C_{k_{h}}$ containing $\beta$ as an interior point and if $\lim _{z \rightarrow \beta} F(z)=F_{h}, z \in C_{k_{h}}, h=1, \cdots, m$, then $\lim _{n \rightarrow \infty} S_{n}(\beta)=\sum_{1}^{m} F_{h} / m$.

The proof may be given by using Lemma 11.2 to show that the conditions in the hypothesis of Theorem 11.2 are satisfied by the numbers $F_{h}$ and by the transforms on $R$ of the function $F(z)$.

We turn now to the question of uniform convergence. By an arc of $\Gamma$, we shall mean a closed arc. A proper subarc of an arc $A$ will be a closed subarc which contains neither end-point of $A$.

Our first theorem is the analogue of Jordan's test.

TheOREM 11.6. Let $\beta$ be a point of $\Gamma$ of multiplicity $m, m \geqq 1$, belonging to the $m$ curves $C_{k_{1}}, C_{k_{2}}, \cdots, C_{k_{m}}$. Let $A_{h}$ be an arc of $C_{k_{h}}$ containing $\beta$ as an interior point, but containing no point of $\Gamma$ of multiplicity greater than one, other 
than $\beta$. If $F(z)$ is single-valued, continuous $\left({ }^{29}\right)$, and of bounded variation on the set $\sum_{h=1}^{m} 1 A_{h}$, then $\lim _{n \rightarrow \infty} S_{n}(z)=F(z)$ uniformly on any proper subarc of $A_{h}$, $h=1, \cdots, m$. If for any given $h, A_{h}=C_{k_{h}}$, then $\lim _{n \rightarrow \infty} S_{n}(z)=F(z)$ uniformly on $C_{k_{h}}$.

We base the proof on Theorem 11.1(b) and on certain properties of the functions $\chi_{p, q}(\theta, \theta+t)$, which we study by means of two lemmas. For future reference, we state these results in a somewhat more precise form than is necessary for present purposes.

Let

$$
\begin{aligned}
& S_{\alpha}(t, \theta)=S(t, \theta)=\left\{\begin{array}{l}
\frac{1}{t} \int_{0}^{t}\left|\frac{\sin \frac{1}{2} \theta}{\sin \frac{1}{2}(\theta+\tau)}\right|^{\alpha} d \tau, \quad 0<\alpha<1, t \neq 0, \\
1, t=0,
\end{array}\right. \\
& \sigma_{\alpha}(t, \theta)=\sigma(t, \theta)=\left\{\begin{array}{l}
\frac{1}{t} \int_{0}^{t}\left|\frac{\theta}{\theta+\tau}\right|^{\alpha} d \tau, \quad 0<\alpha<1, t \neq 0, \\
1, \quad t=0 .
\end{array}\right.
\end{aligned}
$$

LEMma 11.7. The functions $S(t, \theta), V(S ; t), \sigma(t, \theta)$, and $V(\sigma ; t)$ are uniformly bounded, $|\theta| \leqq \frac{1}{2} \pi,|t| \leqq \frac{1}{2} \pi$.

For the proof, we observe first that the function

$$
\left|\frac{\sin \frac{1}{2}(\theta)}{\theta} \cdot \frac{\theta+t}{\sin \frac{1}{2}(\theta+t)}\right|^{\alpha}
$$

and its abolute variation with respect to $t$ are uniformly bounded for $|\theta| \leqq \frac{1}{2} \pi$, $|t| \leqq \frac{1}{2} \pi$, so by Lemma 11.2 , it will suffice to establish the desired result for the function $\sigma(t, \theta)$. The result is trivial for $\theta=0$; henceforth we suppose that $\theta \neq 0$.

$$
\sigma(t, \theta)=\frac{\theta}{t} \int_{0}^{t / \theta} \frac{1}{|1+u|^{\alpha}} d u=\frac{1}{U} \int_{0}^{U} \frac{1}{|1+u|^{\alpha}} d u=f(U),
$$

where $U=t / \theta$. The function $f(U)$ is continuous for all values of $U$, and is a monotone increasing function of $U$ for $U<-1$ and a monotone decreasing function of $U$ for $U>-1$. Thus for any fixed value of $\theta, V(S ; t)$ is equal either to $|\sigma(t, \theta)-\sigma(0, \theta)|$ or to $\sigma(-\theta, \theta)-\sigma(0, \theta)+\sigma(-\theta, \theta)-\sigma(t, \theta)$, and since $\sigma(t, \theta)$ is obviously uniformly bounded for all values of $t$ and $\theta$, so also is $V(S ; t)$. The proof is complete.

\section{Now let}

${ }^{(29)}$ If $F(z)$ is of bounded variation on an arc of $\Gamma$, it can have only simple discontinuities on that arc. It can be shown that such discontinuities are impossible on each of the curves $C_{k}$; therefore this condition of continuity may be replaced by the requirement that $F(z)$ be singlevalued for $z=\beta$. 


$$
X_{p, q}(t, \theta)=\left\{\begin{array}{l}
\frac{1}{t} \int_{0}^{t} \chi_{p, q}(\theta, \theta+\tau) d \tau, t \neq 0, \\
0, t=0,
\end{array} \quad p=1, \cdots, \lambda, q=1, \cdots, \lambda,\right.
$$

and

$$
Y_{p, q}(t, \theta)=\left\{\begin{array}{l}
\frac{1}{t} \int_{0}^{t}\left|\chi_{p, q}(\theta, \theta+\tau)\right| d \tau, t \neq 0, \\
0, t=0,
\end{array} \quad p=1, \cdots, \lambda, q=1, \cdots, \lambda .\right.
$$

Lemma 11.8. If $T$ is any positive number, there exists a number $M(T)$ independent of $\theta$ and $t$ such that $\left|X_{p, q}(t, \theta)\right| \leqq Y_{p, q}(t, \theta) \leqq M(T), V\left(X_{p, q} ; t\right) \leqq M(T)$, $p=1, \cdots, \lambda, q=1, \cdots, \lambda$, for $|t| \leqq T$ and for all values of $\theta$.

The functions $X_{p, q}$ and $Y_{p, q}$ are each periodic functions of $\theta$, so for a given $p$ and $q$ it will suffice to prove the lemma for the values of $\theta$ in a period. Now the numerator of the third member of (10.4) is a polynomial in $\zeta_{q}(\theta)$ and $\zeta_{j}\left(\theta^{\prime}\right), j=1, \cdots, \lambda, j \neq p$, so this numerator is bounded in modulus for all $\theta$ and $\theta^{\prime}$. Therefore, since $\int_{0}^{t}\left|\omega_{q}(\theta+\tau)\right|^{-1} d \tau$ is uniformly bounded for all $\theta$ and for $|t| \leqq T$, so also is $\int_{0}^{t}\left|\chi_{p, q}(\theta, \theta+\tau)\right| d \tau$. Furthermore,

$$
V\left(t \cdot X_{p, q} ; t\right) \leqq \int_{-T}^{T}\left|\chi_{p, q}(\theta, \theta+\tau)\right| d \tau
$$

for $|t| \leqq T$ and for all $\theta$. It follows from these remarks that given any $\delta$, $0<\delta<T$, a positive number $M(\delta)$ independent of $\theta$ exists such that

$\left|X_{p, q}(t, \theta)\right| \leqq Y_{p, q}(t, \theta) \leqq M(\delta), \quad V\left(X_{p, q} ; t\right) \leqq M(\delta), \quad \delta \leqq|t| \leqq T, \quad$ all $\theta$.

We now prove that for each real number $\xi$, there exist positive numbers $M(\xi)$ and $\delta(\xi)$ independent of $t$ and $\theta$ such that $Y_{p, q}(t, \theta) \leqq M(\xi)$ and $V\left(X_{p, q} ; t\right) \leqq M(\xi)$ for $|t| \leqq \delta(\xi)$ and for $\theta$ in the interval $I(\xi): \xi-\delta(\xi) \leqq \theta \leqq \xi$ $+\delta(\xi)$. If $\omega_{q}^{\prime}(\xi) \neq 0$, then it may easily be shown (compare the proof of Theorem 11.2) that both $\left|\chi_{p, q}(\theta, \theta+t)\right|$ and $V\left[\chi_{p, q} ; t\right]$ are uniformly bounded for $t$ suitably restricted and for $\theta$ in some interval containing $\xi$ in its interior. The existence of $\delta(\xi)$ and $M(\xi)$ then follows from Lemma 11.2.

Suppose instead that $\xi \equiv \xi_{j}(\bmod 2 \pi)$ and $\zeta_{q_{1}}(\xi)=\zeta_{q_{2}}(\xi)=\cdots=\zeta_{q_{m}}(\xi)=\beta$. Let $\delta(\xi)=\delta_{j} / 2$. If $p \neq q_{h}, h=1, \cdots, m$, the existence of $M(\xi)$ is again immediate, this time because of Lemma 11.6 (with $\phi(\theta)=1$ ). If $p=q_{H}, 1 \leqq H \leqq m$, and $q=q_{K}, 1 \leqq K \leqq m$, we write

$$
\begin{aligned}
\chi_{p, q}(\theta, \theta+t) & =\frac{\prod_{h}^{\prime}\left(\zeta_{q}(\theta+t)-\zeta_{q_{h}}(\theta)\right)}{\left(\zeta_{q}(\theta+t)-\beta\right)^{m-1}} R(\theta, \theta+t) \\
& =R(\theta, \theta+t) \prod_{h}^{\prime}\left[1-\frac{\zeta_{q_{h}}(\theta)-\beta}{\zeta_{q}(\theta+t)-\beta}\right]
\end{aligned}
$$


where $\prod_{h}^{\prime}=\prod_{h=1}^{m}, h \neq H$, and where $R(\theta, \theta+t)$ is a rational function of $\zeta_{q}(\theta+t)$ with constant coefficients in the denominator, and with numerator coefficients which are polynomials in certain of the functions $\zeta_{p}(\theta)$. Moreover $R(\theta, \theta+t)$, considered as a function of $\zeta_{q}(\theta+t)$, has no poles for $|\theta-\xi| \leqq \frac{1}{2} \delta_{j}$ and $|t| \leqq \frac{1}{2} \delta_{j}$. Consequently, $|R(\theta, \theta+t)|$ and $V(R ; t)$ are uniformly bounded for $\theta$ in $I(\xi)$ and $|t| \leqq \delta(\xi)$. By reference to Lemma 11.2, it is now seen that our problem reduces to one of showing that if we let

$$
\begin{aligned}
& x(t, \theta)=\left\{\begin{array}{l}
\frac{1}{t} \int_{0}^{t} \prod_{h}^{\prime \prime}\left[\frac{\zeta_{q_{h}}(\theta)-\beta}{\zeta_{q}(\theta+\tau)-\beta}\right] d \tau, \quad t \neq 0, \\
1, \quad t=0,
\end{array}\right. \\
& y(t, \theta)=\left\{\begin{array}{l}
\frac{1}{t} \int_{0}^{t} \prod_{h}^{\prime \prime}\left|\frac{\zeta_{q_{h}}(\theta)-\beta}{\zeta_{q}(\theta+\tau)-\beta}\right| d \tau, \quad t \neq 0, \\
1, \quad t=0,
\end{array}\right.
\end{aligned}
$$

where in $\prod_{h}^{\prime \prime}$, the index $h$ assumes $\sigma$ values which are an arbitrary subset (not containing the integer $H$ ) of the first $m$ positive integers-then $y(t, \theta)$ and $V(x ; t)$ are uniformly bounded for $\theta$ in $I(\xi)$ and for $|t| \leqq \delta(\xi)$.

We use (6.5) to write

$$
\begin{aligned}
& x(t, \theta)=\frac{1}{t} \int_{0}^{t}\left|\frac{\sin \frac{1}{2}(\theta-\xi)}{\sin \frac{1}{2}(\theta-\xi+\tau)}\right|^{\sigma / m}\left[e^{(i \sigma / m)(\psi(\theta)-\psi(\theta+\tau))}\right. \\
& \left.\cdot e^{2 \pi i \Delta / m} \frac{\prod_{h}^{\prime \prime} A\left(W_{h}\right)}{[A(\bar{W})]^{\sigma}}\right] d \tau, \quad t \neq 0, \\
& y(t, \theta)=\frac{1}{t} \int_{0}^{t}\left|\frac{\sin \frac{1}{2}(\theta-\xi)}{\sin \frac{1}{2}(\theta-\xi+\tau)}\right|^{\sigma / m} \frac{\left|\prod_{h}^{\prime \prime} A\left(W_{h}\right)\right|}{|A(\bar{W})|^{\sigma}} d \tau, \quad t \neq 0,
\end{aligned}
$$

where $\bar{W}=\left(e^{i(\theta+\tau)}-e^{i \xi}\right)_{K}^{1 / m}$, and $\Delta$ is a constant independent of $\theta$ and $t$. The quantity in brackets in (11.5) is uniformly bounded in modulus for $\theta$ in $I(\xi)$ and for $|\tau| \leqq \delta(\xi)$, and so also is its absolute variation with respect to $\tau$ (compare proof of Theorem 8.4). Lemmas 11.2 and 11.7 therefore permit us to draw the desired conclusions concerning $x(t, \theta)$ and $y(t, \theta)$.

The proof of the lemma may now be completed by an appeal to the HeineBorel theorem.

We now proceed with the proof of Theorem 11.6. Let us suppose (as obviously we may) that the functions $z_{p}(w)$ are numbered in such a way that $z=\zeta_{h}(\theta), \theta_{h} \leqq \theta \leqq \theta_{h}^{\prime}, h=1, \cdots, m$, are the equations respectively of the $\operatorname{arcs} A_{h}$, $h=1, \cdots, m$. We choose the intervals $I_{h}:\left[\theta_{h}, \theta_{h}{ }^{\prime}\right]$ so that each contains the number $\xi$ such that $\zeta_{1}(\xi)=\zeta_{2}(\xi)=\cdots=\zeta_{m}(\xi)=\beta$. Since the numbering of the curves $C_{k_{h}}$ is quite arbitrary, it will evidently suffice to prove that $\lim _{n \rightarrow \infty} s_{1, n}(\theta)=f_{1}(\theta)$ uniformly on the closed interval $I(\delta):\left[\theta_{1}+\delta, \theta_{1}^{\prime}-\delta\right]$, where $\delta$ is any positive number less than the smaller of the two numbers 
$\left(\theta_{1}^{\prime}-\xi\right) / 2$ and $\left(\xi-\theta_{1}\right) / 2$. This will be done by proving the following result.

Lemma 11.9. The hypotheses of Theorem 11.6 imply that $J_{1}\left(t, \theta ; f_{1}(\theta)\right)$ has property $A$ uniformly on $I(\delta)$.

As we remarked in the proof of Theorem 8.4, $f_{h}(\theta)$ is continuous, and of bounded variation on $\theta$ for $\theta_{h} \leqq \theta \leqq \theta_{h}{ }^{\prime}$. We now write $J_{1}\left(t, \theta ; f_{1}(\theta)\right)=\sum_{h=1}^{\lambda}\left(P_{h}+Q_{h}\right)$ where

$$
\begin{aligned}
& P_{h}=\left\{\begin{array}{l}
\frac{1}{t} \int_{0}^{t}\left(f_{h}(\theta+\tau)-f_{1}(\theta)\right) \chi_{1, h}(\theta, \theta+\tau) d \tau, \quad t \neq 0, \\
0, \quad t=0
\end{array}\right. \\
& Q_{h}=\left\{\begin{array}{l}
\frac{1}{t} \int_{0}^{t}\left(f_{h}(\theta-\tau)-f_{1}(\theta)\right) \chi_{1, h}(\theta, \theta-\tau) d \tau, \quad t \neq 0, \\
0, \quad t=0 .
\end{array}\right.
\end{aligned}
$$

It follows immediately from Lemmas 11.8 and 11.2 (in which we let $g(t, \theta)$ $\left.=f_{1}(\theta+t)-f_{1}(\theta)\right)$ that $P_{1}$ and $Q_{1}$ are of bounded variation in $t$ for $\theta$ in $I(\delta)$, and for $0 \leqq t \leqq \delta$, and that $\lim _{t \rightarrow 0} P_{1}=\lim _{t \rightarrow 0} Q_{1}=\lim _{t \rightarrow 0} V\left(P_{1} ; t\right)=\lim _{t \rightarrow 0} V\left(Q_{1} ; t\right)=0$ uniformly for $\theta$ on $I(\delta)$.

Consider now $P_{H}, 2 \leqq H \leqq m$. Given any $\epsilon>0$, there exists a positive number $\delta(\epsilon)<\delta$ independent of $\theta$ and $t$ such that if we write $g(t, \theta)=f_{H}(\theta+t)-f_{1}(\theta)$ $=\left[f_{H}(\theta+t)-f_{H}(\xi)\right]+\left[f_{H}(\xi)-f_{1}(\theta)\right]$, then $|g(t, \theta)|<\epsilon$ and $V(g ; t)<\epsilon$ for $|\theta-\xi| \leqq \delta(\epsilon),|t| \leqq \delta(\epsilon)$. We find from Lemma 11.8 (in which we let $T=\delta$ ) and Lemma 11.2, that $\left|P_{H}\right| \leqq 4 A \epsilon M(\delta)$ and $V\left(P_{H} ; t\right)<4 A \epsilon M(\delta)$ for $(\theta-\xi) \leqq \delta(\epsilon)$, $|t| \leqq \delta(\epsilon)$. On the other hand, if $|t| \leqq \delta(\epsilon) / 2$ and if $\theta_{1} \leqq \theta \leqq \xi-\delta(\epsilon), \xi+\delta(\epsilon)$ $\leqq \theta \leqq \theta_{1}^{\prime}$, then the quantity $\left|\zeta_{H}(\theta+t)-\zeta_{1}(\theta)\right|$ is bounded from zero, and also $\left|f_{1}(\theta)\right|$ is bounded. We then find from Lemma 11.6 (in which we let $T=\delta(\epsilon) / 2$ ) that $P_{H}$ is of bounded variation in $t$ for these values of $t$ and $\theta$, and that furthermore, there exists a positive number $\delta^{\prime}(\epsilon) \leqq \delta(\epsilon) / 2$ such that $\left|P_{H}\right|<\epsilon$ and $V\left(P_{H} ; t\right)<\epsilon$ for $|t| \leqq \delta^{\prime}(\epsilon)$ and for $\theta_{1} \leqq \theta \leqq \xi-\delta(\epsilon), \xi+\delta(\epsilon) \leqq \theta \leqq \theta_{1}^{\prime}$. We have proved that $P_{H}$ has property $A$ uniformly on $I(\delta)$. Moreover, the argument has been stated so as to show simultaneously that $Q_{H}$ has property $\mathcal{A}$ uniformly on $I(\delta)$.

Now $\left|\zeta_{1}(\theta)-\zeta_{h}(\theta+t)\right| \geqq|| \zeta_{h}(\theta+t)-\zeta_{1}(\theta+t)|-| \zeta_{1}(\theta+t)-\zeta_{1}(\theta)||$. If $h>m$, then there exist positive numbers $d$ and $\delta^{\prime \prime} \leqq \delta$ independent of $\theta$ and $t$ such that $\left|\zeta_{h}(\theta+t)-\zeta_{1}(\theta+t)\right|>d$ and $\left|\zeta_{1}(\theta+t)-\zeta_{1}(\theta)\right|<d / 2$ for $\theta$ on $I(\delta)$ and $|t| \leqq \delta^{\prime \prime}$. Therefore, although the point $\zeta_{h}(\theta)$ may lie on the curve $C_{k_{1}}$, nevertheless $\left|\zeta_{1}(\theta)-\zeta_{h}(\theta+t)\right|$ is uniformly bounded from zero for all such values of $\theta$ and $t$. It follows from Lemma 11.6 that $P_{h}$ and $Q_{h}, h>m$, have property $A$ uniformly on $I(\delta)$.

We have now shown that $J_{1}\left(t, \theta ; f_{1}(\theta)\right)$ is the sum of a finite number of functions, each of which has property $\mathcal{A}$ uniformly on $I(\delta)$. It follows at once 
that $J_{1}$ itself has property $\mathcal{A}$ uniformly on $I(\delta)$, and the proof of Lemma 11.9 is complete.

It follows immediately from Theorem 11.1 (b) that $\lim _{n \rightarrow \infty} S_{n}(z)=F(z)$ uniformly on the subarc of $A_{1}$ which is the transform of $I(\delta)$.

If $A_{1}=C_{k_{1}}$, we may replace the interval $I(\delta)$ by the interval $\left[\theta_{1}, \theta_{1}^{\prime}\right]$ in the above argument, where now $\theta_{1}^{\prime}-\theta_{1}=2 \pi \kappa_{k_{1}}$. We then find that $\lim _{n \rightarrow \infty} S_{n}(z)$ $=F(z)$ uniformly on $C_{k_{1}}$. The proof of the theorem is complete.

It is of interest to observe that if $\lim _{n \rightarrow \infty} S_{n}(z)=F(z)$ uniformly on $C_{k}$, then the sequence $\left\{S_{n}(z)\right\}$ converges uniformly in the closed limited Jordan region bounded by $C_{k}$, by the principle of the maximum. It follows in this case that $F(z)$ is uniformly continuous in this closed region $\left({ }^{30}\right)$.

We append two corollaries of Theorem 11.6; the second of these will be stated in terms of the notation introduced in the proof of that theorem.

TheOREM 11.7. If $F(z)$ is continuous $\left.{ }^{31}\right)$ and of bounded variation on $\Gamma$, then $\lim _{n \rightarrow \infty} S_{n}(z)=F(z)$ uniformly on $\bar{D}$.

THEOREM 11.8. If (i) $m>1$, (ii) $F(z)$ is single valued, continuous $\left({ }^{31}\right)$, and of bounded variation on $A_{1}$, (iii) $P_{h}$ and $Q_{h}$ have property $A$ uniformly on $I_{h}$, $h=2, \cdots, m$, then $\lim _{n \rightarrow \infty} S_{n}(z)=F(z)$ uniformly on any proper subarc of $A_{1}$.

The proof of Theorem 11.6 may also be given by a direct consideration of the integral in (10.9), but further lemmas of the Riemann-Lebesgue type are then needed to take care of the terms involving $f_{h}(\theta+\tau), h>1$. Our development has indicated that the tests in Theorem 11.1(a) and (b) include all the other tests of this section. We add the remark that the tests contained in Theorems 11.3 and 11.5 are not comparable.

\section{REFERENCES}

1. G. A. Bliss, Algebraic Functions, New York, 1933.

2. E. Borel, Leçons sur les Fonctions des Variables Réelles, Paris, 1905.

3. T. J. I'A. Bromwich, An Introduction to the Theory of Infinite Series, $2 \mathrm{~d}$ edition, London, 1926.

4. J. H. Curtiss, A note on the Cesàro method of summation, Bulletin of the American Mathematical Society, vol. 43 (1937), pp. 703-708.

5. M. Fekete, Über den Schottkyschen Satz, Journal für die reine und angewandte Mathematik, vol. 165 (1931), pp. 217-224.

6. G. Frobenius, Über die Entwicklung analytischer Funktionen in Reihen, die nach gegebenen Funktionen fortschreiten, ibid., vol. 73 (1871), pp. 1-30.

7. Édouard Goursat, Cours d'Analyse, vol. 2, part 1; English translation by E. R. Hedrick and Otto Dunkel, Boston, 1916.

(30) With reference to this remark, it is perhaps worth while to point out that the existence of a continuous boundary value function in the sense of $\$ 7$ does not by itself imply that $F(z)$ is uniformly continuous in $D$. To draw this inference, additional restrictions are necessary, such as that $F(z) \in H_{\Gamma}$.

(31) See the footnote of Theorem 11.6. 
8. C. G. J. Jacobi, Über Reihenentwicklungen, welche nach den Potenzen eines gegebenen Polynoms fortschreiten, und zu Coefficienten Polynome eines niedereren Grades haben, Journal für die reine und angewandte Mathematik, vol. 53 (1856-1857), pp. 103-126.

9. P. W. Ketchum, On the expansion of a function analytic at distinct points, Bulletin of the American Mathematical Society, vol. 43 (1937), pp. 115-121.

10. A. Kienast, Über die Darstellung der analytischen Funktionen durch Reihen, die nach Potenzen eines Polynoms fortschreiten und Polynome eines niedereren Grades zu Koeffizienten haben, Dissertation, Zurich, 1906.

11. P. Martinotti, Su le serie d'interpolazione, Rendiconti del'Istituto Lombardo, (2), vol. 43 (1910), pp. 391-401.

12. P. Montel, Séries de Polynomes, Paris, 1910, pp. 47-49, 95-97.

13. K. Rieder, Polynomische Entwicklungen von Funktionen einer komplexen Variabeln, Dissertation, Basel, 1911, pp. 66-85.

14. F. Riesz, Über die Randwerte einer analytischen Funktion, Mathematische Zeitschrift, vol. 18 (1923), pp. 87-95.

15. F. and M. Riesz, Über Randwerte einer analytischen Funktion, Quatrième Congrès des Mathematiciens Scandinaves, 1916, pp. 27-44.

16. S. Saks, Theory of the Integral, 2d revised edition, Warsaw, 1937.

17. J. D. Tamarkin, The Theory of Fourier Series, Providence, 1933 (mimeographed notes).

18. E. C. Titchmarsh, The Theory of Functions, Oxford, 1932.

19. J. L. Walsh, Approximation by Polynomials in the Complex Domain, Paris, 1935, pp. $13-14,45-46$.

20. J. L. Walsh, Interpolation and Approximation by Rational Functions in the Complex Domain, American Mathematical Society Colloquium Publications, vol. 20, New York, 1935.

21. J. L. Walsh, Lemniscates and equipotential curves of Green's function, American Mathematical Monthly, vol. 52 (1935), pp. 1-17.

22. A. Zygmund, Trigonometrical Series, Warsaw, 1935.

Cornell University, ITHACA, N. Y. 